doi:10.13108/2015-7-2-115

\title{
ON SPECTRAL AND PSEUDOSPECTRAL FUNCTIONS OF FIRST-ORDER SYMMETRIC SYSTEMS
}

\author{
V.I. MOGILEVSKII
}

\begin{abstract}
We consider first-order symmetric system $J y^{\prime}-B(t) y=\Delta(t) f(t)$ on an interval $\mathcal{I}=[a, b)$ with the regular endpoint $a$. A distribution matrix-valued function $\Sigma(s), s \in \mathbb{R}$, is called a pseudospectral function of such a system if the corresponding Fourier transform is a partial isometry with the minimally possible kernel. The main result is a parametrization of all pseudospectral functions of a given system by means of a Nevanlinna boundary parameter $\tau$. Similar parameterizations for regular systems have earlier been obtained by Arov and Dym, Langer and Textorius, A. Sakhnovich.
\end{abstract}

Keywords: First-order symmetric system, spectral function, pseudospectral function, Fourier transform, characteristic matrix

Mathematics Subject Classification: 34B08,34B40,34L10,47A06,47B25

\section{INTRODUCTION}

Let $H$ and $\widehat{H}$ be finite dimensional Hilbert spaces, let $\mathbb{H}:=H \oplus \widehat{H} \oplus H$ and let [H] be the set of all linear operators in $\mathbb{H}$. We study the first-order symmetric differential system

$$
J y^{\prime}-B(t) y=\lambda \Delta(t) y, \quad t \in \mathcal{I}, \quad \lambda \in \mathbb{C},
$$

where $B(t)=B^{*}(t)$ and $\Delta(t) \geqslant 0$ are $[\mathbb{H}]$-valued functions defined on an interval $\mathcal{I}=[a, b)$, $b \leqslant \infty$, and integrable on each compact subinterval $[a, \beta] \subset \mathcal{I}$ and

$$
J=\left(\begin{array}{ccc}
0 & 0 & -I_{H} \\
0 & i I_{\widehat{H}} & 0 \\
I_{H} & 0 & 0
\end{array}\right): H \oplus \widehat{H} \oplus H \rightarrow H \oplus \widehat{H} \oplus H .
$$

Let $\mathfrak{H}=L_{\Delta}^{2}(\mathcal{I})$ be the Hilbert space of functions $f: \mathcal{I} \rightarrow \mathbb{H}$ such that

$$
\int_{\mathcal{I}}(\Delta(t) f(t), f(t)) d t<\infty
$$

and let $Y_{0}(\cdot, \lambda)$ be the $[\mathbb{H}]$-valued solution of $(1)$ with $Y_{0}(a, \lambda)=I_{\mathbb{H}}$. An $[\mathbb{H}]$-valued distribution function $\Sigma(\cdot)$ is called a spectral function of system (1) if the Fourier transform $V_{\Sigma}: \mathfrak{H} \rightarrow L^{2}(\Sigma ; \mathbb{H})$ given by

$$
\left(V_{\Sigma} f\right)(s)=\widehat{f}(s):=\int_{\mathcal{I}} Y_{0}^{*}(t, s) \Delta(t) f(t) d t, \quad f(\cdot) \in \mathfrak{H}
$$

is an isometry. If $\Sigma(\cdot)$ is a spectral function, then the inverse Fourier transform is defined for each $f \in \mathfrak{H}$ by

$$
f(t)=\int_{\mathcal{I}} Y_{0}(t, s) d \Sigma(s) \widehat{f}(s)
$$

В.И. МОГИЛЕВСКИЙ, О СПЕКТРАЛЬНЫХ И ПСЕВДОСПЕКТРАЛЬНЫХ ФУНКЦИЯХ СИММЕТРИЧЕСКИХ СИСТЕМ ПЕРВОГО ПОРЯДКА.

Поступила 20 октября 2014 г. 
(the integrals in (3) and (4) converge in the norm of $L^{2}(\Sigma ; \mathbb{H})$ and $\mathfrak{H}$, respectively). If the operator $\Delta(t)$ is invertible a.e. on $\mathcal{I}$, then spectral functions of system (1) exist. Otherwise the Fourier transform may have a nontrivial kernel ker $V_{\Sigma}$ and hence the set of spectral functions may be empty [1, 2, 3. The natural generalization of a spectral function to this case is an $[\mathbb{H}]$-valued distribution function $\Sigma(\cdot)$ such that the Fourier transform $V_{\Sigma}$ of the form (3) is a partial isometry. If $\Sigma(\cdot)$ is such a function, then the inverse Fourier transform (4) is valid for each $f \in \mathfrak{H} \ominus \operatorname{ker} V_{\Sigma}$. Therefore, the following problem seems to be interesting:

- To characterize $[\mathbb{H}]$-valued distribution functions $\Sigma(\cdot)$ such that the corresponding Fourier transform $V_{\Sigma}$ is a partial isometry with minimally possible kernel ker $V_{\Sigma}$ and describe these functions in terms of boundary conditions.

In the paper we solve this problem applying the extension theory of symmetric linear relations to symmetric systems. As it is known, system (1) generates the minimal (symmetric) linear relation $T_{\min }$ and the maximal relation $T_{\max }\left(=T_{\min }^{*}\right)$ in $\mathfrak{H}$ (for more details see Sect. 3.1). The domain $\operatorname{dom} T_{\max }$ of relation $T_{\max }$ is the set of all absolutely continuous functions $y \in \mathfrak{H}$ satisfying

$$
J y^{\prime}-B(t) y=\Delta(t) f(t) \quad(\text { a.e. on } \mathcal{I})
$$

with some $f(\cdot) \in \mathfrak{H}$. Moreover, the multivalued part mul $T_{\min }$ of $T_{\min }$ is the set of all $f(\cdot) \in \mathfrak{H}$ such that the solution $y$ of $(5)$ with $y(a)=0$ satisfies $\Delta(t) y(t)=0$ (a.e. on $\mathcal{I}$ ) and $\lim _{t \rightarrow b}(J y(t), z(t))=0, \quad z \in \operatorname{dom} T_{\max }$.

Recall that system (1) is called regular if $\mathcal{I}$ is a compact interval and quasi-regular if for any $\lambda \in \mathbb{C}$ each solution $y$ of (1) belongs to $\mathfrak{H}$. For a quasi-regular (in particular regular) system the integral in (3) converges in the norm of $\mathfrak{H}$ and hence the Fourier transform $\widehat{f}(\cdot)$ of a function $f(\cdot) \in \mathfrak{H}$ does not depend on a choice of a distribution function $\Sigma(\cdot)$. One can easily show that for a quasi-regular system

$$
\operatorname{mul} T_{\min }=\{f \in \mathfrak{H}: \widehat{f}(s)=0, s \in \mathbb{R}\}
$$

and hence mul $T_{\min }$ coincides with the subspace ker $\widehat{U}$ defined in [4, 3].

The following theorem obtained in the paper plays a crucial role in our considerations.

Theorem 1.1. Let $\Sigma(\cdot)$ be an $[\mathbb{H}]$-valued distribution function such that the Fourier transform $V_{\Sigma}$ is a partial isometry from $\mathfrak{H}$ to $L^{2}(\Sigma ; \mathbb{H})$. Then

$$
\operatorname{mul} T_{\min } \subset \operatorname{ker} V_{\Sigma} \text {. }
$$

For quasi-regular systems formula (7) directly follows from (6). Moreover, under the additional condition $\left\|V_{\Sigma} f\right\|=\|f\|, f \in \operatorname{dom} T_{\text {min }}$, Theorem 1.1 can be derived from the results of [2] (see Remark 3.7 below).

The inclusion (7) makes natural the following definition.

Definition 1.2. An $[\mathbb{H}]$-valued distribution function $\Sigma(\cdot)$ is called a pseudospectral function of system (1) if the Fourier transform $V_{\Sigma}$ is a partial isometry with the minimally possible kernel $\operatorname{ker} V_{\Sigma}=\operatorname{mul} T_{\min }$.

We call system (1) absolutely definite if the Lebesgue measure of the set $\{t \in \mathcal{I}: \Delta(t)$ is invertible $\}$ is positive. The main result of the paper is a parametrization of all pseudospectral and spectral functions of absolutely definite system (1) with deficiency indices $n_{ \pm}\left(T_{\min }\right)$ of the minimal relation satisfying $n_{-}\left(T_{\min }\right) \leqslant n_{+}\left(T_{\min }\right)$. Such a parametrization is given by the following theorem. 
Theorem 1.3. Let system (1) be absolutely definite and assume for simplicity that $n_{+}\left(T_{\min }\right)=n_{-}\left(T_{\min }\right)$. Then:

(1) There exist an auxiliary finite-dimensional Hilbert space $\mathcal{H}_{b}$, operator functions $\Omega_{0}(\lambda)(\in[\mathbb{H}]), S(\lambda)\left(\in\left[H \oplus \widehat{H} \oplus \mathcal{H}_{b}, \mathbb{H}\right]\right) \quad$ and a Nevanlinna operator function $M(\lambda)\left(\in\left[H \oplus \widehat{H} \oplus \mathcal{H}_{b}\right]\right), \lambda \in \mathbb{C} \backslash \mathbb{R}$, such that the identities

$$
\begin{gathered}
\Omega_{\tau}(\lambda)=\Omega_{0}(\lambda)+S(\lambda)\left(C_{0}(\lambda)-C_{1}(\lambda) M(\lambda)\right)^{-1} C_{1}(\lambda) S^{*}(\bar{\lambda}), \quad \lambda \in \mathbb{C} \backslash \mathbb{R} \\
\Sigma_{\tau}(s)=\lim _{\delta \rightarrow+0} \lim _{\varepsilon \rightarrow+0} \frac{1}{\pi} \int_{-\delta}^{s-\delta} \operatorname{Im} \Omega_{\tau}(\sigma+i \varepsilon) d \sigma
\end{gathered}
$$

establish a bijective correspondence between all Nevanlinna pairs $\tau=\left\{C_{0}(\lambda), C_{1}(\lambda)\right\}$, $C_{j}(\lambda) \in\left[H \oplus \widehat{H} \oplus \mathcal{H}_{b}\right], j \in\{0,1\}$, satisfying the admissibility conditions

$$
\begin{gathered}
\lim _{y \rightarrow \infty} \frac{1}{i y}\left(C_{0}(i y)-C_{1}(i y) M(i y)\right)^{-1} C_{1}(i y)=0 \\
\lim _{y \rightarrow \infty} \frac{1}{i y} M(i y)\left(C_{0}(i y)-C_{1}(i y) M(i y)\right)^{-1} C_{0}(i y)=0
\end{gathered}
$$

and all pseudospectral functions $\Sigma_{\tau}(\cdot)$ of the system. Moreover, the above statement holds for arbitrary (not necessarily admissible) Nevanlinna pairs $\tau$ if and only if $\lim _{y \rightarrow \infty} \frac{1}{i y} M(i y)=0$ and $\lim _{y \rightarrow \infty} y \cdot \operatorname{Im}(M(i y) h, h)=+\infty, \quad h \neq 0$.

(2) In the case mul $T_{\min }=\{0\}$ (and only in this case) the set of spectral functions is not empty and statement (1) holds for spectral functions.

Note that operator function $M(\lambda)$ in $(8)$ is defined in terms of the boundary values of respective operator solutions of (1) at the endpoints $a$ and $b$, while $\Omega_{0}(\lambda)$ and $S(\lambda)$ are defined in terms of $M(\lambda)$. Observe also that similar to (8), (9) parametrization of $[H \oplus \widehat{H}]$-valued pseudospectral functions corresponding to self-adjoint extensions of $T_{\min }$ can be found in recent works [5, 6].

Existence of pseudospectral functions follows also from the results of [2, 7]. In these papers all pseudospectral functions of regular system (1) are parametrized in the form close to (8), (9). Note that the proof of the results of [2] is not complete (for more details see Remark 3.23).

Recall that system (1) is called a Hamiltonian system if $\widehat{H}=\{0\} .[H]$-valued pseudospectral functions $\Sigma_{H}(\cdot)$ of a Hamiltonian system corresponding to a certain "truncated" Fourier transform are studied in [4, 1, 3]. In the case $H=\mathbb{C}$ existence of a scalar function $\Sigma_{H}(\cdot)$ is proved in [1. A description of all pseudospectral functions $\Sigma_{H}(\cdot)$ of a regular Hamiltonian system is obtained in [4, 3]. Such a description is given in terms of a linear-fractional transform of a Nevanlinna operator pair, which plays a role of a parameter.

Our approach is based on concepts of a boundary triplet for a symmetric relation and the corresponding Weyl functions (see [8, 9, 10, 11, 12, 13, 14] and references therein). In the framework of this approach the operator $M(\lambda)$ in $(8)$ is the Weyl function of an appropriate boundary triplet for $T_{\max }$. Moreover, conditions (10) and 11) are implied by results on $\Pi$ admissibility from [11, 6].

In conclusion note that spectral functions of very general boundary problems were studied in the recent papers [15, 16].

\section{PRELIMINARIES}

2.1. Notations. The following notations will be used throughout the paper: $\mathfrak{H}, \mathcal{H}$ denote Hilbert spaces; $\left[\mathcal{H}_{1}, \mathcal{H}_{2}\right]$ is the set of all bounded linear operators defined on the Hilbert space 
$\mathcal{H}_{1}$ with values in the Hilbert space $\mathcal{H}_{2} ;[\mathcal{H}]:=[\mathcal{H}, \mathcal{H}] ; P_{\mathcal{L}}$ is the orthoprojection in $\mathfrak{H}$ onto the subspace $\mathcal{L} \subset \mathfrak{H} ; \mathbb{C}_{+}\left(\mathbb{C}_{-}\right)$is the upper (lower) half-plane of the complex plane.

Recall that a closed linear relation from $\mathcal{H}_{0}$ to $\mathcal{H}_{1}$ is a closed linear subspace in $\mathcal{H}_{0} \oplus \mathcal{H}_{1}$. The set of all closed linear relations from $\mathcal{H}_{0}$ to $\mathcal{H}_{1}$ (in $\left.\mathcal{H}\right)$ will be denoted by $\widetilde{\mathcal{C}}\left(\mathcal{H}_{0}, \mathcal{H}{ }_{1}\right)(\widetilde{\mathcal{C}}(\mathcal{H}))$. A closed linear operator $T$ from $\mathcal{H}_{0}$ to $\mathcal{H}_{1}$ is identified with its graph $\operatorname{gr} T \in \widetilde{\mathcal{C}}\left(\mathcal{H}_{0}, \mathcal{H}_{1}\right)$.

For a linear relation $T \in \widetilde{\mathcal{C}}\left(\mathcal{H}_{0}, \mathcal{H}_{1}\right)$ we denote by $\operatorname{dom} T, \operatorname{ran} T, \operatorname{ker} T$ and mul $T$ the domain, range, kernel and the multivalued part of $T$ respectively. Recall that mul $T$ ia a subspace in $\mathcal{H}_{1}$ defined by

$$
\operatorname{mul} T:=\left\{h_{1} \in \mathcal{H}_{1}:\left\{0, h_{1}\right\} \in T\right\} .
$$

Clearly, $T \in \widetilde{\mathcal{C}}\left(\mathcal{H}_{0}, \mathcal{H}_{1}\right)$ is an operator if and only if mul $T=\{0\}$. The inverse and adjoint linear relations of $T$ are the relations $T^{-1} \in \widetilde{\mathcal{C}}\left(\mathcal{H}_{1}, \mathcal{H}_{0}\right)$ and $T^{*} \in \widetilde{\mathcal{C}}\left(\mathcal{H}_{1}, \mathcal{H}_{0}\right)$ defined by

$$
\begin{gathered}
T^{-1}=\left\{\left\{h_{1}, h_{0}\right\} \in \mathcal{H}_{1} \oplus \mathcal{H}_{0}:\left\{h_{0}, h_{1}\right\} \in T\right\} \\
T^{*}=\left\{\left\{k_{1}, k_{0}\right\} \in \mathcal{H}_{1} \oplus \mathcal{H}_{0}:\left(k_{0}, h_{0}\right)-\left(k_{1}, h_{1}\right)=0,\left\{h_{0}, h_{1}\right\} \in T\right\} .
\end{gathered}
$$

Recall also that an operator function $\Phi(\cdot): \mathbb{C} \backslash \mathbb{R} \rightarrow[\mathcal{H}]$ is called a Nevanlinna function if it is holomorphic and satisfies $\operatorname{Im} \lambda \cdot \operatorname{Im} \Phi(\lambda) \geqslant 0$ and $\Phi^{*}(\lambda)=\Phi(\bar{\lambda}), \lambda \in \mathbb{C} \backslash \mathbb{R}$.

2.2. Symmetric relations and generalized resolvents. Recall that a linear relation $A \in \widetilde{\mathcal{C}}(\mathfrak{H})$ is called symmetric (self-adjoint) if $A \subset A^{*}$ (resp. $A=A^{*}$ ). For each symmetric relation $A \in \widetilde{\mathcal{C}}(\mathfrak{H})$ the following decompositions hold

$$
\mathfrak{H}=\mathfrak{H}_{0} \oplus \operatorname{mul} A, \quad A=\operatorname{gr} A_{0} \oplus \widehat{\operatorname{mul}} A,
$$

where $\widehat{\operatorname{mul}} A=\{0\} \oplus \operatorname{mul} A$ and $A_{0}$ is a closed symmetric not necessarily densely defined operator in $\mathfrak{H}_{0}$ (the operator part of $A$ ). Moreover, $A=A^{*}$ if and only if $A_{0}=A_{0}^{*}$.

Let $A=A^{*} \in \widetilde{\mathcal{C}}(\mathfrak{H})$, let $\mathcal{B}$ be the Borel $\sigma$-algebra of $\mathbb{R}$ and let $E_{0}(\cdot): \mathcal{B} \rightarrow\left[\mathfrak{H}_{0}\right]$ be the orthogonal spectral measure of $A_{0}$. Then the spectral measure $E_{A}(\cdot): \mathcal{B} \rightarrow[\mathfrak{H}]$ of $A$ is defined as $E_{A}(B)=E_{0}(B) P_{\mathfrak{H}_{0}}, B \in \mathcal{B}$.

Definition 2.1. Let $\widetilde{A}=\widetilde{A}^{*} \in \widetilde{\mathcal{C}}(\widetilde{\mathfrak{H}})$ and let $\mathfrak{H}$ be a subspace in $\widetilde{\mathfrak{H}}$. Relation $\widetilde{A}$ is called $\mathfrak{H}$-minimal if $\overline{\operatorname{span}}\left\{\mathfrak{H},(\widetilde{A}-\lambda)^{-1} \mathfrak{H}: \lambda \in \mathbb{C} \backslash \mathbb{R}\right\}=\widetilde{\mathfrak{H}}$.

Definition 2.2. The relations $T_{j} \in \widetilde{\mathcal{C}}\left(\mathfrak{H}_{j}\right), j \in\{1,2\}$, are said to be unitarily equivalent (by means of a unitary operator $\left.U \in\left[\mathfrak{H}_{1}, \mathfrak{H}_{2}\right]\right)$ if $T_{2}=\widetilde{U} T_{1}$ with $\widetilde{U}=U \oplus U \in\left[\mathfrak{H}_{1}^{2}, \mathfrak{H}_{2}^{2}\right]$.

Let $A \in \widetilde{\mathcal{C}}(\mathfrak{H})$ be a symmetric relation. Recall the following definitions and results.

Definition 2.3. A relation $\widetilde{A}=\widetilde{A}^{*}$ in a Hilbert space $\widetilde{\mathfrak{H}} \supset \mathfrak{H}$ satisfying $A \subset \widetilde{A}$ is called an exit space self-adjoint extension of $A$. Moreover, such an extension $\widetilde{A}$ is called minimal if it is $\mathfrak{H}$-minimal.

In what follows we denote by $\widetilde{\operatorname{Self}}(A)$ the set of all minimal exit space self-adjoint extensions of $A$. Moreover, we denote by $\operatorname{Self}(A)$ the set of all extensions $\widetilde{A}=\widetilde{A}^{*} \in \widetilde{\mathcal{C}}(\mathfrak{H})$ of $A$ (such an extension is called canonical). As is known, for each $A$ one has $\widetilde{\operatorname{Self}}(A) \neq \emptyset$. Moreover, $\operatorname{Self}(A) \neq \emptyset$ if and only if $A$ has equal deficiency indices, in which case $\operatorname{Self}(A) \subset \widetilde{\operatorname{Self}}(A)$.

Definition 2.4. Exit space extensions $\widetilde{A}_{j}=\widetilde{A}_{j}^{*} \in \widetilde{\mathcal{C}}\left(\widetilde{\mathfrak{H}}_{j}\right), j \in\{1,2\}$, of $A$ are called equivalent (with respect to $\mathfrak{H}$ ) if there exists a unitary operator $V \in\left[\widetilde{\mathfrak{H}}_{1} \ominus \mathfrak{H}, \widetilde{\mathfrak{H}}_{2} \ominus \mathfrak{H}\right]$ such that $\widetilde{A}_{1}$ and $\widetilde{A}_{2}$ are unitarily equivalent by means of $U=I_{\mathfrak{H}} \oplus V$. 
Definition 2.5. The operator functions $R(\cdot): \mathbb{C} \backslash \mathbb{R} \rightarrow[\mathfrak{H}]$ and $F(\cdot): \mathbb{R} \rightarrow[\mathfrak{H}]$ are called a generalized resolvent and a spectral function of $A$ respectively if there exists an exit space extension $\widetilde{A}$ of $A$ (in a certain Hilbert space $\widetilde{\mathfrak{H}} \supset \mathfrak{H}$ ) such that

$$
\begin{gathered}
R(\lambda)=P_{\mathfrak{H}}(\widetilde{A}-\lambda)^{-1}\lceil\mathfrak{H}, \quad \lambda \in \mathbb{C} \backslash \mathbb{R} \\
F(t)=P_{\mathfrak{H}} E((-\infty, t)) \uparrow \mathfrak{H}, \quad t \in \mathbb{R} .
\end{gathered}
$$

Here $P_{\mathfrak{H}}$ is the orthoprojection in $\widetilde{\mathfrak{H}}$ onto $\mathfrak{H}$ and $E(\cdot)$ is the spectral measure of $\widetilde{A}$.

In the case $\widetilde{A} \in \operatorname{Self}(A)$ identity $(12)$ defines the canonical resolvent $R(\lambda)=(\widetilde{A}-\lambda)^{-1}$ of $\mathrm{A}$.

Proposition 2.6. Each generalized resolvent $R(\lambda)$ of $A$ is generated by some (minimal) extension $\widetilde{A} \in \widetilde{\operatorname{Self}}(A)$. Moreover, the extensions $\widetilde{A}_{1}, \widetilde{A_{2}} \in \widetilde{\operatorname{Self}}(A)$ inducing the same generalized resolvent $R(\cdot)$ are equivalent.

In the sequel we suppose that a generalized resolvent $R(\cdot)$ and a spectral function $F(\cdot)$ are generated by an extension $\widetilde{A} \in \widetilde{\operatorname{Self}}(A)$. Moreover, we identify equivalent extensions. Then by Proposition 2.6 identity $(12)$ gives a bijective correspondence between generalized resolvents $R(\lambda)$ and extensions $\widetilde{A} \in \widehat{\operatorname{Self}}(A)$, so that each $\widetilde{A} \in \widetilde{\operatorname{Self}}(A)$ is uniquely defined by the corresponding generalized resolvent $(12)$ (spectral function $(13)$ ).

It follows from (12) and (13) that the generalized resolvent $R(\cdot)$ and the spectral function $F(\cdot)$ generated by an extension $\widetilde{A} \in \widetilde{\operatorname{Self}}(A)$ are related by

$$
R(\lambda)=\int_{\mathbb{R}} \frac{d F(t)}{t-\lambda}, \quad \lambda \in \mathbb{R}
$$

Moreover, setting $\widetilde{\mathfrak{H}}_{0}=\widetilde{\mathfrak{H}} \ominus$ mul $\widetilde{A}$ one gets from 13 that

$$
F(\infty)\left(:=s-\lim _{t \rightarrow+\infty} F(t)\right)=P_{\mathfrak{H}} P_{\widetilde{\mathfrak{H}}_{0}}\lceil\mathfrak{H}
$$

2.3. The spaces $\mathcal{L}^{2}(\Sigma ; \mathcal{H})$ and $L^{2}(\Sigma ; \mathcal{H})$. Let $\mathcal{H}$ be a finite dimensional Hilbert space. A non-decreasing operator function $\Sigma(\cdot): \mathbb{R} \rightarrow[\mathcal{H}]$ is called a distribution function if it is left continuous and satisfies $\Sigma(0)=0$.

Theorem 2.7. [17, ch. 3.15], [18] Let $\Sigma(\cdot): \mathbb{R} \rightarrow[\mathcal{H}]$ be a distribution function. Then:

(1) There exist a scalar measure $\sigma$ on Borel sets of $\mathbb{R}$ and a function $\Psi: \mathbb{R} \rightarrow[\mathcal{H}]$ (uniquely defined by $\sigma$ up to $\sigma$-a.e. $)$ such that $\Psi(s) \geqslant 0 \sigma$-a.e. on $\mathbb{R}, \sigma([\alpha, \beta))<\infty$ and $\Sigma(\beta)-\Sigma(\alpha)=\int_{[\alpha, \beta)} \Psi(s) d \sigma(s)$ for any finite interval $[\alpha, \beta) \subset \mathbb{R}$.

(2) The set $\mathcal{L}^{2}(\Sigma ; \mathcal{H})$ of all Borel-measurable functions $f(\cdot): \mathbb{R} \rightarrow \mathcal{H}$ satisfying

$$
\|f\|_{\mathcal{L}^{2}(\Sigma ; \mathcal{H})}^{2}=\int_{\mathbb{R}}(d \Sigma(s) f(s), f(s)):=\int_{\mathbb{R}}(\Psi(s) f(s), f(s))_{\mathcal{H}} d \sigma(s)<\infty
$$

is a semi-Hilbert space with the semi-scalar product

$$
(f, g)_{\mathcal{L}^{2}(\Sigma ; \mathcal{H})}=\int_{\mathbb{R}}(d \Sigma(s) f(s), g(s)):=\int_{\mathbb{R}}(\Psi(s) f(s), g(s))_{\mathcal{H}} d \sigma(s), \quad f, g \in \mathcal{L}^{2}(\Sigma ; \mathcal{H}) .
$$

Moreover, different measures $\sigma$ from statement $(1)$ give rise to the same space $\mathcal{L}^{2}(\Sigma ; \mathcal{H})$.

Definition 2.8. [17, 18] The Hilbert space $L^{2}(\Sigma ; \mathcal{H})$ is a Hilbert space of all equivalence classes in $\mathcal{L}^{2}(\Sigma ; \mathcal{H})$ with respect to the seminorm $\|\cdot\|_{\mathcal{L}^{2}(\Sigma ; \mathcal{H})}$. 
In the following we denote by $\pi_{\Sigma}$ the quotient map from $\mathcal{L}^{2}(\Sigma ; \mathcal{H})$ onto $L^{2}(\Sigma ; \mathcal{H})$. Moreover, we denote by $\mathcal{L}_{\text {loc }}^{2}(\Sigma ; \mathcal{H})$ the set of all functions $g \in \mathcal{L}^{2}(\Sigma ; \mathcal{H})$ with the compact support and we put $L_{l o c}^{2}(\Sigma ; \mathcal{H}):=\pi_{\Sigma} \mathcal{L}_{l o c}^{2}(\Sigma ; \mathcal{H})$.

With a distribution function $\Sigma(\cdot)$ one associates the multiplication operator $\Lambda=\Lambda_{\Sigma}$ in $L^{2}(\Sigma ; \mathcal{H})$ defined by

$$
\begin{gathered}
\operatorname{dom} \Lambda_{\Sigma}=\left\{\tilde{f} \in L^{2}(\Sigma ; \mathcal{H}): s f(s) \in \mathcal{L}^{2}(\Sigma ; \mathcal{H}) \text { for some (and hence for all) } f(\cdot) \in \widetilde{f}\right\} \\
\Lambda_{\Sigma} \widetilde{f}=\pi_{\Sigma}(s f(s)), \quad \widetilde{f} \in \operatorname{dom} \Lambda_{\Sigma}, \quad f(\cdot) \in \widetilde{f} .
\end{gathered}
$$

As is known, $\Lambda_{\Sigma}^{*}=\Lambda_{\Sigma}$ and the spectral measure $E_{\Sigma}$ of $\Lambda_{\Sigma}$ is given by

$$
E_{\Sigma}(B) \tilde{f}=\pi_{\Sigma}\left(\chi_{B}(\cdot) f(\cdot)\right), \quad B \in \mathcal{B}, \quad \widetilde{f} \in L^{2}(\Sigma ; \mathcal{H}), \quad f(\cdot) \in \widetilde{f}
$$

where $\chi_{B}(\cdot)$ is the indicator of the Borel set $B$.

Let $\mathcal{K}, \mathcal{K}^{\prime}$ and $\mathcal{H}$ be finite dimensional Hilbert spaces and let $\Sigma(s)(\in[\mathcal{H}])$ be a distribution function. For Borel functions $Y(s)(\in[\mathcal{H}, \mathcal{K}])$ and $g(s)(\in \mathcal{H})$ we let

$$
\int_{\mathbb{R}} Y(s) d \Sigma(s) g(s):=\int_{\mathbb{R}} Y(s) \Psi(s) g(s) d \sigma(s)(\in \mathcal{K})
$$

where $\sigma$ and $\Psi(\cdot)$ are defined in Theorem 2.7, (1).

2.4. The classes $\widetilde{R}_{+}\left(\mathcal{H}_{0}, \mathcal{H}_{1}\right)$ and $\widetilde{R}(\mathcal{H})$. Let $\mathcal{H}_{0}$ be a Hilbert space, let $\mathcal{H}_{1}$ be a subspace in $\mathcal{H}_{0}$ and let $\tau=\left\{\tau_{+}, \tau_{-}\right\}$be a collection of holomorphic functions $\tau_{ \pm}(\cdot): \mathbb{C}_{ \pm} \rightarrow \widetilde{\mathcal{C}}\left(\mathcal{H}_{0}, \mathcal{H}_{1}\right)$. In the paper we systematically deal with collections $\tau=\left\{\tau_{+}, \tau_{-}\right\}$of the special class $\widetilde{R}_{+}\left(\mathcal{H}_{0}, \mathcal{H}_{1}\right)$. Definition and detailed characterization of this class can be found in our paper [6] (see also [19, 20, 5], where the notation $\widetilde{R}\left(\mathcal{H}_{0}, \mathcal{H}_{1}\right)$ were used instead of $\left.\widetilde{R}_{+}\left(\mathcal{H}_{0}, \mathcal{H}_{1}\right)\right)$. If $\operatorname{dim} \mathcal{H}_{1}<\infty$, then according to [6] the collection $\tau=\left\{\tau_{+}, \tau_{-}\right\} \in \widetilde{R}_{+}\left(\mathcal{H}_{0}, \mathcal{H}_{1}\right)$ admits the representation

$$
\tau_{+}(\lambda)=\left\{\left(C_{0}(\lambda), C_{1}(\lambda)\right) ; \mathcal{H}_{0}\right\}, \quad \lambda \in \mathbb{C}_{+} ; \quad \tau_{-}(\lambda)=\left\{\left(D_{0}(\lambda), D_{1}(\lambda)\right) ; \mathcal{H}_{1}\right\}, \quad \lambda \in \mathbb{C}_{-}
$$

by means of two pairs of holomorphic operator functions

$$
\left(C_{0}(\lambda), C_{1}(\lambda)\right): \mathcal{H}_{0} \oplus \mathcal{H}_{1} \rightarrow \mathcal{H}_{0}, \quad \lambda \in \mathbb{C}_{+}, \quad \text { and }\left(D_{0}(\lambda), D_{1}(\lambda)\right): \mathcal{H}_{0} \oplus \mathcal{H}_{1} \rightarrow \mathcal{H}_{1}, \quad \lambda \in \mathbb{C}_{-}
$$

(more precisely, by equivalence classes of such pairs). Identities (18) mean that

$$
\begin{array}{ccc}
\tau_{+}(\lambda)=\left\{\left\{h_{0}, h_{1}\right\} \in \mathcal{H}_{0} \oplus \mathcal{H}_{1}: C_{0}(\lambda) h_{0}+C_{1}(\lambda) h_{1}=0\right\}, & \lambda \in \mathbb{C}_{+} \\
\tau_{-}(\lambda)=\left\{\left\{h_{0}, h_{1}\right\} \in \mathcal{H}_{0} \oplus \mathcal{H}_{1}: D_{0}(\lambda) h_{0}+D_{1}(\lambda) h_{1}=0\right\}, & \lambda \in \mathbb{C}_{-} .
\end{array}
$$

In [6] the class $\widetilde{R}_{+}\left(\mathcal{H}_{0}, \mathcal{H}_{1}\right)$ was characterized both in terms of $\widetilde{\mathcal{C}}\left(\mathcal{H}_{0}, \mathcal{H}_{1}\right)$-valued functions $\tau_{ \pm}(\cdot)$ and in terms of operator functions $C_{j}(\cdot)$ and $D_{j}(\cdot), j \in\{0,1\}$, from 18 .

If $\mathcal{H}_{1}=\mathcal{H}_{0}=: \mathcal{H}$, then the class $\widetilde{R}(\mathcal{H}):=\widetilde{R}_{+}(\mathcal{H}, \mathcal{H})$ coincides with the well-known class of Nevanlinna $\widetilde{\mathcal{C}}(\mathcal{H})$-valued functions $\tau(\cdot)$ (see, for instance, [11]). In this case the collection (18) turns into the Nevanlinna pair

$$
\tau(\lambda)=\left\{\left(C_{0}(\lambda), C_{1}(\lambda)\right) ; \mathcal{H}\right\}, \quad \lambda \in \mathbb{C} \backslash \mathbb{R},
$$

with $C_{0}(\lambda), C_{1}(\lambda) \in[\mathcal{H}]$. Recall also that the subclass $\widetilde{R}^{0}(\mathcal{H}) \subset \widetilde{R}(\mathcal{H})$ is defined as the set of all $\tau(\cdot) \in \widetilde{R}(\mathcal{H})$ such that $\tau(\lambda) \equiv \theta\left(=\theta^{*}\right), \lambda \in \mathbb{C} \backslash \mathbb{R}$. This implies that $\tau(\cdot) \in \widetilde{R}^{0}(\mathcal{H})$ if and only if

$$
\tau(\lambda) \equiv\left\{\left(C_{0}, C_{1}\right) ; \mathcal{H}\right\}, \quad \lambda \in \mathbb{C} \backslash \mathbb{R},
$$

with some operators $C_{0}, C_{1} \in[\mathcal{H}]$ satisfying $\operatorname{Im}\left(C_{1} C_{0}^{*}\right)=0$ and $0 \in \rho\left(C_{0} \pm i C_{1}\right.$ ) (for more details see e.g. [5, Remark 2.5]). 
2.5. Boundary triplets and Weyl functions. Here we recall definitions of a boundary triplet and the corresponding Weyl function of a symmetric relation following [8, 9, 13, 12, 10, 14, 21, 6].

Let $A$ be a closed symmetric linear relation in the Hilbert space $\mathfrak{H}$, let $\mathfrak{N}_{\lambda}(A)=\operatorname{ker}\left(A^{*}-\lambda\right)$ $(\lambda \in \mathbb{C})$ be a defect subspace of $A$, let $\widehat{\mathfrak{N}}_{\lambda}(A)=\left\{\{f, \lambda f\}: f \in \mathfrak{N}_{\lambda}(A)\right\}$ and let $n_{ \pm}(A):=\operatorname{dim} \mathfrak{N}_{\lambda}(A) \leqslant \infty, \quad \lambda \in \mathbb{C}_{ \pm}$, be deficiency indices of $A$.

Next, assume that $\mathcal{H}_{0}$ is a Hilbert space, $\mathcal{H}_{1}$ is a subspace in $\mathcal{H}_{0}$ and $\mathcal{H}_{2}:=\mathcal{H}_{0} \ominus \mathcal{H}_{1}$, so that $\mathcal{H}_{0}=\mathcal{H}_{1} \oplus \mathcal{H}_{2}$. Denote by $P_{j}$ the orthoprojection in $\mathcal{H}_{0}$ onto $\mathcal{H}_{j}, j \in\{1,2\}$.

Definition 2.9. A collection $\Pi_{+}=\left\{\mathcal{H}_{0} \oplus \mathcal{H}_{1}, \Gamma_{0}, \Gamma_{1}\right\}$, where $\Gamma_{j}: A^{*} \rightarrow \mathcal{H}_{j}, j \in\{0,1\}$, are linear mappings, is called a boundary triplet for $A^{*}$, if the mapping $\Gamma: \widehat{f} \rightarrow\left\{\Gamma_{0} \widehat{f}, \Gamma_{1} \widehat{f}\right\}, \widehat{f} \in A^{*}$, from $A^{*}$ into $\mathcal{H}_{0} \oplus \mathcal{H}_{1}$ is surjective and the following Green's identity holds

$$
\left(f^{\prime}, g\right)-\left(f, g^{\prime}\right)=\left(\Gamma_{1} \widehat{f}, \Gamma_{0} \widehat{g}\right)_{\mathcal{H}_{0}}-\left(\Gamma_{0} \widehat{f}, \Gamma_{1} \widehat{g}\right)_{\mathcal{H}_{0}}+i\left(P_{2} \Gamma_{0} \widehat{f}, P_{2} \Gamma_{0} \widehat{g}\right)_{\mathcal{H}_{2}}
$$

holds for all $\widehat{f}=\left\{f, f^{\prime}\right\}, \widehat{g}=\left\{g, g^{\prime}\right\} \in A^{*}$.

According to 21 a boundary triplet $\Pi_{+}=\left\{\mathcal{H}_{0} \oplus \mathcal{H}_{1}, \Gamma_{0}, \Gamma_{1}\right\}$ for $A^{*}$ exists if and only if $n_{-}(A) \leqslant n_{+}(A)$, in which case $\operatorname{dim} \mathcal{H}_{1}=n_{-}(A)$ and $\operatorname{dim} \mathcal{H}_{0}=n_{+}(A)$.

Proposition 2.10. [21] Let $\Pi_{+}=\left\{\mathcal{H}_{0} \oplus \mathcal{H}_{1}, \Gamma_{0}, \Gamma_{1}\right\}$ be a boundary triplet for $A^{*}$. Then the identities

$$
\begin{gathered}
\Gamma_{1}\left\lceil\widehat{\mathfrak{N}}_{\lambda}(A)=M_{+}(\lambda) \Gamma_{0}\left\lceil\widehat{\mathfrak{N}}_{\lambda}(A), \quad \lambda \in \mathbb{C}_{+}\right.\right. \\
\left(\Gamma_{1}+i P_{2} \Gamma_{0}\right) \uparrow \widehat{\mathfrak{N}}_{\lambda}(A)=M_{-}(\lambda) P_{1} \Gamma_{0}\left\lceil\widehat{\mathfrak{N}}_{\lambda}(A), \quad \lambda \in \mathbb{C}_{-}\right.
\end{gathered}
$$

well define the (holomorphic) operator functions $M_{+}(\cdot) \quad: \quad \mathbb{C}_{+} \quad \rightarrow \quad\left[\mathcal{H}_{0}, \mathcal{H}_{1}\right]$ and $M_{-}(\cdot): \mathbb{C}_{-} \rightarrow\left[\mathcal{H}_{1}, \mathcal{H}_{0}\right]$ satisfying $M_{+}^{*}(\bar{\lambda})=M_{-}(\lambda), \lambda \in \mathbb{C}_{-}$.

Definition 2.11. 21] The operator functions $M_{ \pm}(\cdot)$ defined in Proposition 2.10 are called the Weyl functions corresponding to the boundary triplet $\Pi_{+}$.

Theorem 2.12. [21] Let $A$ be a closed symmetric linear relation in $\mathfrak{H}$, let $\Pi_{+}=\left\{\mathcal{H}_{0} \oplus \mathcal{H}_{1}, \Gamma_{0}, \Gamma_{1}\right\}$ be a boundary triplet for $A^{*}$ and let $M_{+}(\cdot)$ be the corresponding Weyl function. If $\tau=\left\{\tau_{+}, \tau_{-}\right\} \in \widetilde{R}_{+}\left(\mathcal{H}_{0}, \mathcal{H}_{1}\right)$ is a collection of holomorphic pairs (18), then for every $g \in \mathfrak{H}$ and $\lambda \in \mathbb{C} \backslash \mathbb{R}$ the abstract boundary value problem

$$
\begin{array}{cc}
\{f, \lambda f+g\} \in A^{*} & \\
C_{0}(\lambda) \Gamma_{0}\{f, \lambda f+g\}-C_{1}(\lambda) \Gamma_{1}\{f, \lambda f+g\}=0, & \lambda \in \mathbb{C}_{+} \\
D_{0}(\lambda) \Gamma_{0}\{f, \lambda f+g\}-D_{1}(\lambda) \Gamma_{1}\{f, \lambda f+g\}=0, & \lambda \in \mathbb{C}_{-}
\end{array}
$$

has a unique solution $f=f(g, \lambda)$ and the identity $R(\lambda) g:=f(g, \lambda)$ defines a generalized resolvent $R(\lambda)=R_{\tau}(\lambda)$ of $A$. Moreover, $0 \in \rho\left(\tau_{+}(\lambda)+M_{+}(\lambda)\right)$ and the following KreinNaimark formula for resolvents is valid:

$$
R_{\tau}(\lambda)=\left(A_{0}-\lambda\right)^{-1}-\gamma_{+}(\lambda)\left(\tau_{+}(\lambda)+M_{+}(\lambda)\right)^{-1} \gamma_{-}^{*}(\bar{\lambda}), \quad \lambda \in \mathbb{C}_{+}
$$

Conversely, for each generalized resolvent $R(\lambda)$ of $A$ there exists a unique $\tau \in \widetilde{R}_{+}\left(\mathcal{H}_{0}, \mathcal{H}_{1}\right)$ such that $R(\lambda)=R_{\tau}(\lambda)$ and, consequently, identity (24) is valid.

Remark 2.13. It follows from Theorem 2.12 that the boundary value problem (21)-(23) as well as formula for resolvents (24) give a parametrization of all generalized resolvents

$$
R(\lambda)=R_{\tau}(\lambda)=P_{\mathfrak{H}}\left(\widetilde{A}^{\tau}-\lambda\right)^{-1}\lceil\mathfrak{H}, \quad \lambda \in \mathbb{C} \backslash \mathbb{R},
$$


and, consequently, all extensions $\widetilde{A}=\widetilde{A^{\tau}} \in \widetilde{\operatorname{Self}}(A)$ of $A$ by means of an abstract boundary parameter $\tau \in \widetilde{R}_{+}\left(\mathcal{H}_{0}, \mathcal{H}_{1}\right)$.

Definition 2.14. An extension $\widetilde{A} \in \widetilde{\operatorname{Self}}(A)(\widetilde{A} \in \operatorname{Self}(A))$ is referred to the class $\widetilde{\operatorname{Self}}_{0}(A)$ (resp. $\left.\operatorname{Self}_{0}(A)\right)$ if mul $\widetilde{A}=\operatorname{mul} A$.

Theorem 2.15. Let under the assumptions of Theorem 2.12 $\tau=\left\{\tau_{+}, \tau_{-}\right\} \in \widetilde{R}_{+}\left(\mathcal{H}_{0}, \mathcal{H}_{1}\right)$ be a collection of holomorphic pairs (18) and let $\widetilde{A}^{\tau} \in \widetilde{\operatorname{Self}}(A)$ be the corresponding extension of A (see Remark 2.13). Then:

(1) Identities

$$
\begin{gathered}
\Phi_{\tau}(\lambda):=P_{1}\left(C_{0}(\lambda)-C_{1}(\lambda) M_{+}(\lambda)\right)^{-1} C_{1}(\lambda), \quad \lambda \in \mathbb{C}_{+} \\
\widehat{\Phi}_{\tau}(\lambda)=M_{+}(\lambda)\left(C_{0}(\lambda)-C_{1}(\lambda) M_{+}(\lambda)\right)^{-1} C_{0}(\lambda) \uparrow \mathcal{H}_{1}, \quad \lambda \in \mathbb{C}_{+}
\end{gathered}
$$

define holomorphic $\left[\mathcal{H}_{1}\right]$-valued functions $\Phi_{\tau}(\cdot)$ and $\widehat{\Phi}_{\tau}(\cdot)$ on $\mathbb{C}_{+}$satisfying $\operatorname{Im} \Phi_{\tau}(\lambda) \geqslant 0$ and $\operatorname{Im} \widehat{\Phi}_{\tau}(\lambda) \geqslant 0, \lambda \in \mathbb{C}_{+}$. Hence there exist strong limits

$$
\begin{gathered}
\mathcal{B}_{\tau}:=s-\lim _{y \rightarrow+\infty} \frac{1}{i y} P_{1}\left(C_{0}(i y)-C_{1}(i y) M_{+}(i y)\right)^{-1} C_{1}(i y) \\
\widehat{\mathcal{B}}_{\tau}:=s-\lim _{y \rightarrow+\infty} \frac{1}{i y} M_{+}(i y)\left(C_{0}(i y)-C_{1}(i y) M_{+}(i y)\right)^{-1} C_{0}(i y)\left\lceil\mathcal{H}_{1}\right.
\end{gathered}
$$

(2) The inclusion $\widetilde{A}^{\tau} \in \widetilde{\operatorname{Self}}_{0}(A)$ holds if and only if $\mathcal{B}_{\tau}=\widehat{\mathcal{B}}_{\tau}=0$

Proof. Statement (1) for $\Phi_{\tau}(\lambda)$ was proved in [6, Theorem 4.8].

Next assume that

$$
\begin{aligned}
& C_{0}(\lambda)=\left(C_{01}(\lambda), C_{02}(\lambda)\right): \mathcal{H}_{1} \oplus \mathcal{H}_{2} \rightarrow \mathcal{H}_{0}, \quad D_{0}(\lambda)=\left(D_{01}(\lambda), D_{02}(\lambda)\right): \mathcal{H}_{1} \oplus \mathcal{H}_{2} \rightarrow \mathcal{H}_{1} \\
& M_{+}(\lambda)=\left(M(\lambda), N_{+}(\lambda)\right): \mathcal{H}_{1} \oplus \mathcal{H}_{2} \rightarrow \mathcal{H}_{1}, \quad M_{-}(\lambda)=\left(M(\lambda), N_{-}(\lambda)\right)^{\top}: \mathcal{H}_{1} \rightarrow \mathcal{H}_{1} \oplus \mathcal{H}_{2}
\end{aligned}
$$

are the block-matrix representations of $C_{0}(\lambda), D_{0}(\lambda)$ and $M_{ \pm}(\lambda)$. Moreover,let

$$
\begin{gathered}
\widehat{C}_{0}(\lambda)=\left(C_{1}(\lambda), C_{02}(\lambda)\right): \mathcal{H}_{1} \oplus \mathcal{H}_{2} \rightarrow \mathcal{H}_{0} ; \quad \widehat{C}_{1}(\lambda)=-C_{01}(\lambda), \quad \lambda \in \mathbb{C}_{+} \\
\widehat{M}_{+}(\lambda)=\left(-M^{-1}(\lambda),-M^{-1}(\lambda) N_{+}(\lambda)\right): \mathcal{H}_{1} \oplus \mathcal{H}_{2} \rightarrow \mathcal{H}_{1}, \quad \lambda \in \mathbb{C}_{+}
\end{gathered}
$$

Then according to [6], the identities

$$
\widehat{\Phi}_{\tau}(\lambda):=P_{1}\left(\widehat{C}_{0}(\lambda)-\widehat{C}_{1}(\lambda) \widehat{M}_{+}(\lambda)\right)^{-1} \widehat{C}_{1}(\lambda), \quad \lambda \in \mathbb{C}_{+} ; \quad \widehat{\Phi}_{\tau}(\lambda):=\widehat{\Phi}_{\tau}^{*}(\bar{\lambda}), \quad \lambda \in \mathbb{C}_{-}
$$

define a Nevanlinna function $\widehat{\Phi}_{\tau}(\cdot): \mathbb{C} \backslash \mathbb{R} \rightarrow\left[\mathcal{H}_{1}\right]$ (i.e., a holomorphic function $\widehat{\Phi}_{\tau}(\cdot)$ such that $\operatorname{Im} \lambda \cdot \operatorname{Im} \widehat{\Phi}_{\tau}(\lambda) \geqslant 0$ and $\left.\widehat{\Phi}_{\tau}^{*}(\lambda)=\widehat{\Phi}_{\tau}(\bar{\lambda}), \lambda \in \mathbb{C} \backslash \mathbb{R}\right)$. The immediate checking shows that

$$
\left(P_{2}-M_{+}(\lambda)\right)^{-1}=-M^{-1}(\lambda) P_{1}-M^{-1}(\lambda) N_{+}(\lambda) P_{2}+P_{2}
$$

and, consequently, $P_{1}\left(P_{2}-M_{+}(\lambda)\right)^{-1}=\widehat{M}_{+}(\lambda)$ (here $M_{+}(\lambda)$ is considered as the operator in $\left.\mathcal{H}_{0}\right)$. This and 30 imply that for each $\lambda \in \mathbb{C}_{+}$

$$
\begin{gathered}
\widehat{\Phi}_{\tau}(\lambda)=-P_{1}\left(C_{1}(\lambda) P_{1}+C_{02}(\lambda) P_{2}+C_{01}(\lambda) P_{1}\left(P_{2}-M_{+}(\lambda)\right)^{-1}\right)^{-1} C_{01}(\lambda)= \\
-P_{1}\left(P_{2}-M_{+}(\lambda)\right)\left(\left(C_{1}(\lambda) P_{1}+C_{02}(\lambda) P_{2}\right)\left(P_{2}-M_{+}(\lambda)\right)+C_{01}(\lambda) P_{1}\right)^{-1} C_{01}(\lambda)= \\
M_{+}(\lambda)\left(C_{02}(\lambda) P_{2}-C_{1}(\lambda) M_{+}(\lambda)+C_{01}(\lambda) P_{1}\right)^{-1} C_{01}(\lambda)= \\
M_{+}(\lambda)\left(C_{0}(\lambda)-C_{1}(\lambda) M_{+}(\lambda)\right)^{-1} C_{0}(\lambda)\left\lceil\mathcal{H}_{1} .\right.
\end{gathered}
$$

Thus the restriction of $\widehat{\Phi}_{\tau}(\cdot)$ on $\mathbb{C}_{+}$admits representation (27), which yields statement (1) for $\widehat{\Phi}_{\tau}(\lambda)$. 
It was shown in [6] that the second identity in (30) can be written as

$$
\widehat{\Phi}_{\tau}(\lambda):=M(\lambda)\left(D_{01}(\lambda)-D_{1}(\lambda) M(\lambda)-i D_{02}(\lambda) N_{-}(\lambda)\right)^{-1} D_{01}(\lambda), \quad \lambda \in \mathbb{C}_{-}
$$

Therefore, by (29) one has

$$
\begin{gathered}
\widehat{\mathcal{B}}_{\tau}=s-\lim _{y \rightarrow+\infty} \frac{1}{i y} \widehat{\Phi}_{\tau}(i y)=s-\lim _{y \rightarrow-\infty} \frac{1}{i y} \widehat{\Phi}_{\tau}(i y)= \\
s-\lim _{y \rightarrow-\infty} \frac{1}{i y} M(i y)\left(D_{01}(i y)-D_{1}(i y) M(i y)-i D_{02}(i y) N_{-}(i y)\right)^{-1} D_{01}(i y) .
\end{gathered}
$$

Now statement (2) follows from [6, Theorem 4.9].

Remark 2.16. (1) If $\mathcal{H}_{0}=\mathcal{H}_{1}:=\mathcal{H}$, then the boundary triplet in the sense of Definition 2.9 turns into the boundary triplet $\Pi=\left\{\mathcal{H}, \Gamma_{0}, \Gamma_{1}\right\}$ for $A^{*}$ in the sense of [12, 9]. In this case $n_{+}(A)=n_{-}(A)(=\operatorname{dim} \mathcal{H})$ and $M_{ \pm}(\cdot)$ turn into the Weyl function $M(\cdot): \mathbb{C} \backslash \mathbb{R} \rightarrow[\mathcal{H}]$ introduced in [10, 14]. Moreover, in this case $M(\cdot)$ is a Nevanlinna operator function.

In the sequel a boundary triplet $\Pi=\left\{\mathcal{H}, \Gamma_{0}, \Gamma_{1}\right\}$ in the sense of [12, 9] will be called an ordinary boundary triplet for $A^{*}$.

(2) Let $n_{+}(A)=n_{-}(A)$, let $\Pi=\left\{\mathcal{H}, \Gamma_{0}, \Gamma_{1}\right\}$ be an ordinary boundary triplet for $A^{*}$ and let $M(\cdot)$ be the corresponding Weyl function. Then an abstract boundary parameter $\tau$ in Theorem 2.12 is a Nevanlinna operator pair $\tau \in \widetilde{R}(\mathcal{H})$ of the form $(19)$ and identities 28 and (29) become

$$
\begin{gathered}
\mathcal{B}_{\tau}=s-\lim _{y \rightarrow \infty} \frac{1}{i y}\left(C_{0}(i y)-C_{1}(i y) M(i y)\right)^{-1} C_{1}(i y) \\
\widehat{\mathcal{B}}_{\tau}=s-\lim _{y \rightarrow \infty} \frac{1}{i y} M(i y)\left(C_{0}(i y)-C_{1}(i y) M(i y)\right)^{-1} C_{0}(i y) .
\end{gathered}
$$

Note that for this case Theorem 2.15 was proved in [11, 22].

\section{PSEUdospectral AND SPECTRAL FUnCTIONS OF SYMMETRIC SYSTEMS}

3.1. Symmetric systems. Let $H$ and $\widehat{H}$ be finite dimensional Hilbert spaces, let

$$
H_{0}:=H \oplus \widehat{H}, \quad \mathbb{H}:=H_{0} \oplus H=H \oplus \widehat{H} \oplus H
$$

and let $J \in[\mathbb{H}]$ be operator (2). A first order symmetric system of differential equations on an interval $\mathcal{I}=[a, b\rangle,-\infty<a<b \leqslant \infty$, (with the regular endpoint $a$ ) is of the form

$$
J y^{\prime}(t)-B(t) y(t)=\lambda \Delta(t) y, \quad t \in \mathcal{I}, \quad \lambda \in \mathbb{C},
$$

where $B(\cdot)$ and $\Delta(\cdot)$ are the $[\mathbb{H}]$-valued functions on $\mathcal{I}$ integrable on each compact interval $[a, \beta] \subset \mathcal{I}$ and such that $B(t)=B^{*}(t)$ and $\Delta(t) \geqslant 0$ (a.e. on $\mathcal{I}$ ).

An absolutely continuous function $y: \mathcal{I} \rightarrow \mathbb{H}$ is a solution of (34) if identity (34) holds a.e. on $\mathcal{I}$. An operator function $Y(\cdot, \lambda): \mathcal{I} \rightarrow[\mathcal{K}, \mathbb{H}]$ is an operator solution of equation (34) if $y(t)=Y(t, \lambda) h$ is a solution of this equation for every $h \in \mathcal{K}$ (here $\mathcal{K}$ is a Hilbert space with $\operatorname{dim} \mathcal{K}<\infty)$.

The following lemma will be useful in the sequel.

Lemma 3.1. Let $\mathcal{K}$ be a finite dimensional Hilbert space, let $Y(\cdot, \cdot): \mathcal{I} \times \mathbb{R} \rightarrow[\mathcal{K}, \mathbb{H}]$ be an operator function such that $Y(\cdot, s)$ is a solution of (34) and $Y(a, \cdot)$ is a continuous function on $\mathbb{R}$ and let $\Sigma(\cdot): \mathbb{R} \rightarrow[\mathcal{K}]$ be a distribution function. Then for each function $g \in \mathcal{L}_{\text {loc }}^{2}(\Sigma ; \mathcal{K})$ the identity

$$
f(t)=\int_{\mathbb{R}} Y(t, s) d \Sigma(s) g(s), \quad t \in \mathcal{I}
$$


defines an absolutely continuous function $f(\cdot)$ such that

$$
f^{\prime}(t)=-J \int_{\mathbb{R}}(B(t)+s \Delta(t)) Y(t, s) d \Sigma(s) g(s) \quad(\text { a.e.on } \mathcal{I}) .
$$

Proof. In accordance with (17), identity (35) means

$$
f(t)=\int_{\mathbb{R}} Y(t, s) \Psi(s) g(s) d \sigma(s), \quad t \in \mathcal{I},
$$

where $\Psi$ and $\sigma$ are defined in Theorem 2.7, (1). Since $Y(t, s)$ satisfies

$$
Y(t, s)=Y(a, s)-J \int_{[a, t)}(B(u)+s \Delta(u)) Y(u, s) d u, \quad t \in \mathcal{I}
$$

it follows that $Y(\cdot, \cdot)$ is a continuous function on $\mathcal{I} \times \mathbb{R}$. Moreover, one can easily prove that $\int_{\mathbb{R}}\|\Psi(s) g(s)\| d \sigma(s)<\infty$. Therefore, the integral in (37) exists and

$$
\int_{[a, t) \times \mathbb{R}}\|(B(u)+s \Delta(u)) Y(u, s) \Psi(s) g(s)\| d u d \sigma(s)<\infty .
$$

It follows from $(39)$ and the Fubini theorem that

$$
\begin{gathered}
\int_{\mathbb{R}}\left(\int_{[a, t)}(B(u)+s \Delta(u)) Y(u, s) \Psi(s) g(s) d u\right) d \sigma(s)= \\
\int_{[a, t)}\left(\int_{\mathbb{R}}(B(u)+s \Delta(u)) Y(u, s) \Psi(s) g(s) d \sigma(s)\right) d u .
\end{gathered}
$$

Now combining (37) with $(38)$ and taking 40 into account, one gets

$$
f(t)=C-J \int_{[a, t)}\left(\int_{\mathbb{R}}(B(u)+s \Delta(u)) Y(u, s) \Psi(s) g(s) d \sigma(s)\right) d u,
$$

where $C=\int_{\mathbb{R}} Y(a, s) \Psi(s) g(s) d \sigma(s)$. Hence 36 holds.

Denote by $\mathcal{L}_{\Delta}^{2}(\mathcal{I})$ the semi-Hilbert space of Borel measurable functions $f(\cdot): \mathcal{I} \rightarrow \mathbb{H}$ such that $\int_{\mathcal{I}}(\Delta(t) f(t), f(t))_{\mathbb{H}} d t<\infty$ and let $\mathfrak{H}:=L_{\Delta}^{2}(\mathcal{I})$ be the Hilbert space of all equivalence classes in $\mathcal{L}_{\Delta}^{2}(\mathcal{I})$ [17, Chapter 13.5]. Denote also by $\pi_{\Delta}$ the quotient map from $\mathcal{L}_{\Delta}^{2}(\mathcal{I})$ onto $L_{\Delta}^{2}(\mathcal{I})$.

For each system (34) the identities

$$
\begin{aligned}
& \mathcal{T}_{\max }=\left\{\{y, f\} \in\left(\mathcal{L}_{\Delta}^{2}(\mathcal{I})\right)^{2}: y\right. \text { is absolutely continuous and } \\
& \left.\qquad J y^{\prime}(t)-B(t) y(t)=\Delta(t) f(t) \text { a.e. on } \mathcal{I}\right\}
\end{aligned}
$$

and $T_{\max }=\left(\pi_{\Delta} \oplus \pi_{\Delta}\right) \mathcal{T}_{\max }$ define the linear relations $\mathcal{T}_{\max }$ in $\mathcal{L}_{\Delta}^{2}(\mathcal{I})$ and $T_{\max }$ in $\mathfrak{H}$. Moreover, the identity

$$
[y, z]_{b}:=\lim _{t \uparrow b}(J y(t), z(t)), \quad y, z \in \operatorname{dom} \mathcal{T}_{\max } .
$$

well defines the skew-Hermitian bilinear form $[\cdot, \cdot]_{b}$ on dom $\mathcal{T}_{\text {max }}$. By using this form one defines the relations $\mathcal{T}_{a}$ in $\mathcal{L}_{\Delta}^{2}(\mathcal{I})$ and $T_{\min }$ in $\mathfrak{H}$ via

$$
\mathcal{T}_{a}=\left\{\{y, f\} \in \mathcal{T}_{\max }: y(a)=0 \text { and }[y, z]_{b}=0 \text { for every } z \in \operatorname{dom} \mathcal{T}_{\max }\right\}
$$

and $T_{\min }=\left(\pi_{\Delta} \oplus \pi_{\Delta}\right) \mathcal{T}_{a}$. It turns out that $T_{\min }$ is a closed symmetric linear relation in $\mathfrak{H}$ with finite deficiency indices $n_{ \pm}\left(T_{\min }\right)$ and $T_{\min }^{*}=T_{\max }$ (see [23] for regular and [1, 24, 25, 26] for general systems). The relations $T_{\min }$ and $T_{\max }$ are called the minimal and maximal relations respectively.

The following assertion is immediate from definitions of $T_{\min }$ and $T_{\max }$. 
Assertion 3.2. (1) The multivalued part mul $T_{\min }$ of the minimal relation $T_{\min }$ is the set of all $\tilde{f} \in L_{\Delta}^{2}(\mathcal{I})$ such that for some (and hence for all) $f \in \widetilde{f}$ the solution $y$ of the equation

$$
J y^{\prime}-B(t) y=\Delta(t) f(t), \quad t \in \mathcal{I}
$$

with $y(a)=0$ satisfies $\Delta(t) y(t)=0$ (a.e on $\mathcal{I}$ ) and $[y, z]_{b}=0, z \in \operatorname{dom} \mathcal{T}_{\max }$.

(2) The identity mul $T_{\min }=\operatorname{mul} T_{\max }$ holds if and only if for each function $y \in \operatorname{dom} \mathcal{T}_{\max }$ the identity $\Delta(t) y(t)=0$ (a.e. on $\mathcal{I})$ yields $y(a)=0$ and $[y, z]_{b}=0, z \in \operatorname{dom} \mathcal{T}_{\max }$.

3.2. $q$-pseudospectral and spectral functions. Denote by $\mathfrak{H}_{b}$ the set of all $\tilde{f} \in \mathfrak{H}$ with the following property: there exists $\beta_{\tilde{f}} \in \mathcal{I}$ such that for some (and hence for all) function $f \in \tilde{f}$ the identity $\Delta(t) f(t)=0$ holds a.e. on $\left(\beta_{\widetilde{f}}, b\right)$. Moreover, denote by $Y_{0}(\cdot, \lambda)$ the $[\mathbb{H}]$-valued operator solution of (34) satisfying $Y_{0}(a, \lambda)=I_{\mathbb{H}}$. With each $\tilde{f} \in \mathfrak{H}_{b}$ we associate the function $\widehat{f}(\cdot): \mathbb{R} \rightarrow \mathbb{H}$ given by

$$
\widehat{f}(s)=\int_{\mathcal{I}} Y_{0}^{*}(t, s) \Delta(t) f(t) d t, \quad f(\cdot) \in \widetilde{f} .
$$

By using the well-known properties of the solution $Y_{0}(\cdot, \lambda)$, one can easily prove that $\widehat{f}(\cdot)$ is a continuous (and even holomorphic) function on $\mathbb{R}$.

Recall that an operator $V \in\left[\mathfrak{H}_{1}, \mathfrak{H}_{2}\right]$ is called a partial isometry if $\|V f\|=\|f\|$ for all $f \in \mathfrak{H}_{1} \ominus \operatorname{ker} V$.

Definition 3.3. A distribution function $\Sigma(\cdot): \mathbb{R} \rightarrow[\mathbb{H}]$ will be called a $q$-pseudospectral function of the system (34) if $\widehat{f} \in \mathcal{L}^{2}(\Sigma ; \mathbb{H})$ for all $\tilde{f} \in \mathfrak{H}_{b}$ and the operator $V_{b} \widetilde{f}:=\pi_{\Sigma} \widehat{f}, \widetilde{f} \in \mathfrak{H}_{b}$, admits a continuation to a partial isometry $V=V_{\Sigma} \in\left[\mathfrak{H} ; L^{2}(\Sigma ; \mathbb{H})\right]$.

The operator $V=V_{\Sigma}$ will be called the Fourier transform corresponding to $\Sigma(\cdot)$.

Clearly, if $\Sigma(\cdot)$ is a $q$-pseudospectral function, then for each $f(\cdot) \in \mathcal{L}_{\Delta}^{2}(\mathcal{I})$ there exists a unique $\widetilde{g}\left(=V_{\Sigma} \pi_{\Delta} f\right) \in L^{2}(\Sigma ; \mathbb{H})$ such that for each function $g(\cdot) \in \widetilde{g}$ one has

$$
\lim _{\beta \uparrow b}|| g(\cdot)-\left.\int_{[a, \beta)} Y_{0}^{*}(t, \cdot) \Delta(t) f(t) d t\right|_{\mathcal{L}^{2}(\Sigma ; \mathbb{H})}=0 .
$$

Proposition 3.4. Let $\Sigma(\cdot)$ be a q-pseudospectral function and let $V=V_{\Sigma}$ be the corresponding Fourier transform. Then for each $\widetilde{g} \in L_{\text {loc }}^{2}(\Sigma ; \mathbb{H})$ the function

$$
f_{\widetilde{g}}(t):=\int_{\mathbb{R}} Y_{0}(t, s) d \Sigma(s) g(s), \quad g(\cdot) \in \widetilde{g}
$$

belongs to $\mathcal{L}_{\Delta}^{2}(\mathcal{I})$ and $V^{*} \widetilde{g}=\pi_{\Delta} f_{\widetilde{g}}(\cdot)$. Therefore,

$$
V^{*} \widetilde{g}=\pi_{\Delta}\left(\int_{\mathbb{R}} Y_{0}(\cdot, s) d \Sigma(s) g(s)\right), \quad \widetilde{g} \in L^{2}(\Sigma ; \mathbb{H}), \quad g(\cdot) \in \widetilde{g},
$$

where the integral converges in the seminorm of $\mathcal{L}_{\Delta}^{2}(\mathcal{I})$.

Proof. According to Lemma $3.1 f_{\widetilde{g}}(\cdot)$ is a continuous $\mathbb{H}$-valued function on $\mathcal{I}$ and by (17)

$$
f_{\widetilde{g}}(t)=\int_{\mathbb{R}} Y_{0}(t, s) \Psi(s) g(s) d \sigma(s), \quad g(\cdot) \in \widetilde{g},
$$

where $\sigma$ and $\Psi$ are defined in Theorem 2.7, (1).

Let $f_{*}(\cdot) \in \mathcal{L}_{\Delta}^{2}(\mathcal{I})$ be a function such that $\pi_{\Delta} f_{*}(\cdot)=V^{*} \widetilde{g}$. Moreover, let $h \in \mathbb{H}$, let $\delta \subset \mathcal{I}$ be a compact interval and let $f(t)=\chi_{\delta}(t) h\left(\in \mathcal{L}_{\Delta}^{2}(\mathcal{I})\right)$. We show that

$$
\int_{\mathcal{I}}\left(f(t), \Delta(t) f_{\widetilde{g}}(t)\right)_{\mathbb{H}} d t=\int_{\mathcal{I}}\left(f(t), \Delta(t) f_{*}(t)\right)_{\mathbb{H}} d t
$$


In view of 43$)$ one has

$$
\int_{\mathcal{I}}\left(f(t), \Delta(t) f_{\widetilde{g}}(t)\right)_{\mathbb{H}} d t=\int_{\mathcal{I}}\left(\int_{\mathbb{R}}\left(\Delta(t) f(t), Y_{0}(t, s) \Psi(s) g(s)\right)_{\mathbb{H}} d \sigma(s)\right) d t .
$$

Since $Y_{0}(\cdot, \cdot)$ is a continuous function on $\mathcal{I} \times \mathbb{R}$, it follows that

$$
\int_{\mathcal{I} \times \mathbb{R}}\left|\left(\Delta(t) f(t), Y_{0}(t, s) \Psi(s) g(s)\right)_{\mathbb{H}}\right| d t d \sigma(s)<\infty .
$$

Therefore, by the Fubini theorem one has

$$
\begin{gathered}
\int_{\mathcal{I}}\left(\int_{\mathbb{R}}\left(\Delta(t) f(t), Y_{0}(t, s) \Psi(s) g(s)\right)_{\mathbb{H}} d \sigma(s)\right) d t= \\
\int_{\mathbb{R}}\left(\int_{\mathcal{I}}\left(\Delta(t) f(t), Y_{0}(t, s) \Psi(s) g(s)\right)_{\mathbb{H}} d t\right) d \sigma(s)= \\
\int_{\mathbb{R}}\left(\int_{\mathcal{I}}\left(\Psi(s) Y_{0}^{*}(t, s) \Delta(t) f(t), g(s)\right)_{\mathbb{H}} d t\right) d \sigma(s)= \\
\int_{\mathbb{R}}\left(\Psi(s) \int_{\mathcal{I}} Y_{0}^{*}(t, s) \Delta(t) f(t) d t, g(s)\right)_{\mathbb{H}} d \sigma(s)=\left(V \pi_{\Delta} f, \widetilde{g}\right)_{L^{2}(\Sigma ; \mathbb{H})}= \\
\left(\pi_{\Delta} f, V^{*} \widetilde{g}\right)_{\mathfrak{H}}=\int_{\mathcal{I}}\left(f(t), \Delta(t) f_{*}(t)\right) d t .
\end{gathered}
$$

Combining these relations with (45) one gets identity (44).

It follows from (44) that $\Delta(t) f_{\widetilde{g}}(t)=\Delta(t) f_{*}(t)$ (a.e. on $\mathcal{I}$ ). Hence $f_{\widetilde{g}}(\cdot) \in \mathcal{L}_{\Delta}^{2}(\mathcal{I})$ and $\pi_{\Delta} f_{\widetilde{g}}(\cdot)=\pi_{\Delta} f_{*}(\cdot)=V^{*} \widetilde{g}$.

Let $V_{\Sigma}$ be the Fourier transform corresponding to the $q$-pseudospectral function $\Sigma(\cdot)$ and let $\mathfrak{H}_{0}^{\prime}=\mathfrak{H} \ominus \operatorname{ker} V_{\Sigma}, L_{0}=V_{\Sigma} \mathfrak{H}\left(=V_{\Sigma} \mathfrak{H}_{0}^{\prime}\right)$ and $L_{0}^{\perp}=L^{2}(\Sigma ; \mathbb{H}) \ominus L_{0}$. Then

$$
\mathfrak{H}=\operatorname{ker} V_{\Sigma} \oplus \mathfrak{H}_{0}^{\prime}, \quad L^{2}(\Sigma ; \mathbb{H})=L_{0} \oplus L_{0}^{\perp} .
$$

Assume also that

$$
\widetilde{\mathfrak{H}}_{0}^{\prime}:=\mathfrak{H}_{0}^{\prime} \oplus L_{0}^{\perp}, \quad \widetilde{\mathfrak{H}}:=\overbrace{\operatorname{ker} V_{\Sigma} \oplus \mathfrak{H}_{0}^{\prime}}^{\mathfrak{H}} \oplus L_{0}^{\perp}=\mathfrak{H} \oplus L_{0}^{\perp}=\operatorname{ker} V_{\Sigma} \oplus \widetilde{\mathfrak{H}}_{0}^{\prime}
$$

and let $\widetilde{V}^{\prime} \in\left[\widetilde{\mathfrak{H}}_{0}^{\prime}, L^{2}(\Sigma ; \mathbb{H})\right]$ be a unitary operator of the form

$$
\widetilde{V}^{\prime}=\left(V_{\Sigma}\left\lceil\mathfrak{H}_{0}^{\prime}, I_{L_{0}^{\perp}}\right): \mathfrak{H}_{0}^{\prime} \oplus L_{0}^{\perp} \rightarrow L^{2}(\Sigma ; \mathbb{H}),\right.
$$

where $I_{L_{0}^{\perp}}$ is an embedding operator from $L_{0}^{\perp}$ to $L^{2}(\Sigma ; \mathbb{H})$. Since $\mathfrak{H} \subset \widetilde{\mathfrak{H}}$, one may consider $T_{\text {min }}$ as a linear relation in $\widetilde{\mathfrak{H}}$.

Lemma 3.5. Let $\Sigma(\cdot)$ be a q-pseudospectral function of the system (34) and let $\widetilde{V}^{\prime}$ be a unitary operator 48 . Moreover, let $\left(T_{\min }\right)_{\widetilde{\mathfrak{H}}}^{*} \in \widetilde{\mathcal{C}}(\widetilde{\mathfrak{H}})$ be a linear relation adjoint to $T_{\min }$ in $\widetilde{\mathfrak{H}}$ and let $\Lambda=\Lambda_{\Sigma}$ be the multiplication operator in $L^{2}(\Sigma ; \mathbb{H})$. Then the identities

$$
\widetilde{f}=\left(\widetilde{V}^{\prime}\right)^{*} \widetilde{g}, \quad \widetilde{T}_{0} \widetilde{f}=\left(\widetilde{V}^{\prime}\right)^{*} \Lambda \widetilde{g}, \quad \widetilde{g} \in \operatorname{dom} \Lambda
$$

define a self-adjoint operator $\widetilde{T}_{0}$ in $\widetilde{\mathfrak{H}}_{0}^{\prime}$ such that $\widetilde{T}_{0} \subset\left(T_{\min }\right)_{\widetilde{\mathfrak{H}}}^{*}$.

Proof. It is easily seen that $\left(T_{\min }\right)_{\mathfrak{\mathfrak { H }}}^{*}=T_{\max } \oplus\left(L_{0}^{\perp}\right)^{2}$. Moreover, in view of 48) one has

$$
\left(\widetilde{V}^{\prime}\right)^{*} \widetilde{g}=V_{\Sigma}^{*} \widetilde{g}+P_{L_{0}^{\perp}} \widetilde{g}, \quad \widetilde{g} \in L^{2}(\Sigma ; \mathbb{H})
$$


Therefore, (49) can be written as

$$
\widetilde{f}=V_{\Sigma}^{*} \widetilde{g}+P_{L_{0}^{\perp}} \widetilde{g}, \quad \widetilde{T}_{0} \widetilde{f}=V_{\Sigma}^{*} \Lambda \widetilde{g}+P_{L_{0}^{\perp}} \Lambda \widetilde{g}, \quad \widetilde{g} \in \operatorname{dom} \Lambda .
$$

Thus to prove the inclusion $\widetilde{T}_{0} \subset\left(T_{\min }\right)_{\widetilde{\mathfrak{H}}}^{*}$ it is sufficient to show that $\left\{V_{\Sigma}^{*} \widetilde{g}, V_{\Sigma}^{*} \Lambda \widetilde{g}\right\} \in T_{\max }$ for all $\widetilde{g} \in \operatorname{dom} \Lambda$.

Let $\widetilde{g} \in \operatorname{dom} \Lambda, g(\cdot) \in \widetilde{g}$ and let $E(\cdot)=E_{\Sigma}(\cdot)$ be the spectral measure of $\Lambda$. Then by (16) and (15) for each compact interval $\delta \subset \mathbb{R}$ one has $E(\delta) \widetilde{g}=\pi_{\Sigma}\left(\chi_{\delta}(\cdot) g(\cdot)\right)$ and $\Lambda E(\delta) \widetilde{g}=\pi_{\Sigma}\left(s \chi_{\delta}(s) g(s)\right)$. Therefore, according to Proposition $3.4 V_{\Sigma}^{*} E(\delta) \widetilde{g}=\pi_{\Delta} y(\cdot)$ and $V_{\Sigma}^{*} \Lambda E(\delta) \widetilde{g}=\pi_{\Delta} f(\cdot)$, where

$$
y(t)=\int_{\mathbb{R}} Y_{0}(t, s) d \Sigma(s) \chi_{\delta}(s) g(s), \quad f(t)=\int_{\mathbb{R}} s Y_{0}(t, s) d \Sigma(s) \chi_{\delta}(s) g(s) .
$$

It follows from Lemma 3.1 that $y(\cdot)$ is absolutely continuous and

$$
y^{\prime}(t)=-J \int_{\mathbb{R}}(B(t)+s \Delta(t)) Y_{0}(t, s) d \Sigma(s) \chi_{\delta}(s) g(s) \quad(\text { a.e.on } \mathcal{I}) .
$$

Therefore,

$$
J y^{\prime}(t)-B(t) y(t)=\Delta(t) \int_{\mathbb{R}} s Y_{0}(t, s) d \Sigma(s) \chi_{\delta}(s) g(s)=\Delta(t) f(t) \quad(\text { a.e.on } \mathcal{I})
$$

and, consequently, $\{y, f\} \in \mathcal{T}_{\max }$. Hence $\left\{V_{\Sigma}^{*} E(\delta) \widetilde{g}, V_{\Sigma}^{*} \Lambda E(\delta) \widetilde{g}\right\}\left(=\left\{\pi_{\Delta} y(\cdot), \pi_{\Delta} f(\cdot)\right\}\right) \in T_{\max }$ and passage to the limit when $\delta \rightarrow \mathbb{R}$ yields the required inclusion $\left\{V_{\Sigma}^{*} \widetilde{g}, V_{\Sigma}^{*} \Lambda \widetilde{g}\right\} \in T_{\max }$.

Theorem 3.6. For each q-pseudospectral function $\Sigma(\cdot)$ of the system (34) the corresponding Fourier transform $V_{\Sigma}$ satisfies

$$
\operatorname{mul} T_{\min } \subset \operatorname{ker} V_{\Sigma}
$$

(for mul $T_{\min }$ see Assertion 3.2. (1)).

Proof. Let $\widetilde{T}_{0}=\widetilde{T}_{0}^{*}$ be the operator in $\widetilde{\mathfrak{H}}_{0}^{\prime}$ defined in Lemma 3.5 and let $\left(\widetilde{T}_{0}\right)_{\widetilde{\mathfrak{H}}^{\prime}}^{*}$ be the linear relation adjoint to $\widetilde{T}_{0}$ in $\widetilde{\mathfrak{H}}^{\prime}$. Then $\left(\widetilde{T}_{0}\right)_{\mathfrak{H}^{\prime}}^{*}=\widetilde{T}_{0} \oplus\left(\operatorname{ker} V_{\Sigma}\right)^{2}$ and the inclusion $\widetilde{T}_{0} \subset\left(T_{\min }\right)_{\mathfrak{\mathfrak { H }}}^{*}$ yields

$$
T_{\min } \subset \widetilde{T}_{0} \oplus\left(\operatorname{ker} V_{\Sigma}\right)^{2} .
$$

Let $n \in \operatorname{mul} T_{\text {min }}$. Then $\{0, n\} \in T_{\min }$ and by (51) $\{0, n\} \in \widetilde{T}_{0} \oplus\left(\text { ker } V_{\Sigma}\right)^{2}$. Therefore, there exist $f \in \operatorname{dom} \widetilde{T}_{0}$ and $g, g^{\prime} \in \operatorname{ker} V_{\Sigma}$ such that

$$
f+g=0, \quad \widetilde{T}_{0} f+g^{\prime}=n .
$$

Since $f \in \widetilde{\mathfrak{H}}_{0}^{\prime}, g \in \operatorname{ker} V_{\Sigma}$ and $\widetilde{\mathfrak{H}}_{0}^{\prime} \perp \operatorname{ker} V_{\Sigma}($ see 47$)$ ), it follows that $f=g=0$. Therefore, $\widetilde{T}_{0} f=0$ and hence $n=g^{\prime} \in \operatorname{ker} V_{\Sigma}$. This yields the inclusion (50).

Remark 3.7. According to [2, Lemma 5], the identity

$$
\Phi_{s} f=\int_{\mathcal{I}} Y_{0}^{*}(t, s) \Delta(t) f(t) d t, \quad \tilde{f} \in \operatorname{dom} T_{\min } \cap \tilde{H}_{b}, \quad s \in \mathbb{R}
$$

defines a directing mapping $\Phi$ of $T_{\min }$ in the sense of [2]. By using this fact and Theorem 1 from [2] one can prove the inclusion (50) for $q$-pseudospectral functions $\Sigma(\cdot)$ satisfying the additional condition $\left\|V_{\Sigma} \widetilde{f}\right\|=\|\widetilde{f}\|, \widetilde{f} \in \operatorname{dom} T_{\min }$.

Definition 3.8. A $q$-pseudospectral function $\Sigma(\cdot)$ of the system (34) will be called a pseudospectral function if the corresponding Fourier transform $V_{\Sigma}$ satisfies $\operatorname{ker} V_{\Sigma}=\operatorname{mul} T_{\min }$. 
Definition 3.9. A distribution function $\Sigma(\cdot): \mathbb{R} \rightarrow[\mathbb{H}]$ will be called a spectral function of the system (34) if for every $\widetilde{f} \in \mathfrak{H}_{b}$ the inclusion $\widehat{f} \in \mathcal{L}^{2}(\Sigma ; \mathbb{H})$ holds and the Parseval identity $\|\widehat{f}\|_{\mathcal{L}^{2}(\Sigma ; \mathbb{H})}=\|\widetilde{f}\|_{\mathfrak{H}}$ is valid (for $\widehat{f}$ see 420$)$.

It follows from Theorem 3.6 that a pseudospectral function is a $q$-pseudospectral function $\Sigma(\cdot)$ with the minimally possible ker $V_{\Sigma}$. Moreover, the same theorem yields the following assertion.

Assertion 3.10. A distribution function $\Sigma(\cdot): \mathbb{R} \rightarrow[\mathbb{H}]$ is a spectral function of the system (34) if and only if it is a pseudospectral function with $\operatorname{ker} V_{\Sigma}\left(=\operatorname{mul} T_{\min }\right)=\{0\}$ (that is, with the isometry $V_{\Sigma}$ ).

In the following we put $\mathfrak{H}_{0}:=\mathfrak{H} \ominus \operatorname{mul} T_{\text {min }}$, so that

$$
\mathfrak{H}=\operatorname{mul} T_{\min } \oplus \mathfrak{H}_{0} .
$$

Moreover, for a pseudospectral function $\Sigma(\cdot)$ we denote by $V_{0}=V_{0, \Sigma}$ the isometry from $\mathfrak{H}_{0}$ to $L^{2}(\Sigma ; \mathbb{H})$ given by

$$
V_{0, \Sigma}:=V_{\Sigma} \uparrow \mathfrak{H}_{0}
$$

Clearly, $V_{\Sigma}$ admits the representation

$$
V_{\Sigma}=\left(0, V_{0, \Sigma}\right): \operatorname{mul} T_{\min } \oplus \mathfrak{H}_{0} \rightarrow L^{2}(\Sigma ; \mathbb{H})
$$

3.3. Pseudospectral functions and extensions of the minimal relation. Recall that system (34) is called definite if for some (and hence for all) $\lambda \in \mathbb{C}$ there exists only the trivial solution $y=0$ of this system satisfying $\Delta(t) y(t)=0$ a.e. in $\mathcal{I}$. We also introduce the following definition.

Definition 3.11. System (34) will be called absolutely definite if the Lebesgue measure of the set $\{t \in \mathcal{I}: \Delta(t)$ is invertible $\}$ is positive.

Remark 3.12. (1) Clearly, each absolutely definite system is definite. Moreover, one can easily construct definite, but not absolutely definite system (34) (even with $B(t) \equiv 0$ and continuous $\Delta(t))$.

(2) It is known (see e.g. [24]) that the maximal relation $T_{\max }$ induced by the definite symmetric system (34) possesses the following property: for any $\{\widetilde{y}, \widetilde{f}\} \in T_{\max }$ there exists a unique absolutely continuous function $y \in \mathcal{L}_{\Delta}^{2}(\mathcal{I})$ such that $y \in \widetilde{y}$ and $\{y, f\} \in \mathcal{T}_{\max }$ for any $f \in \tilde{f}$. Below we associate such a function $y$ with each pair $\{\widetilde{y}, \widetilde{f}\} \in T_{\max }$.

Similarly to [5, Proposition 6.9] one proves the following proposition.

Proposition 3.13. Let $\Sigma(\cdot)$ be a q-pseudospectral function of the definite system and let $L_{0}$ be a subspace in $L^{2}(\Sigma ; \mathbb{H})$ given by $L_{0}=V_{\Sigma} \mathfrak{H}$. Then the multiplication operator $\Lambda_{\Sigma}$ is $L_{0}$-minimal (in the sense of Definition 2.1).

For a Hilbert space $\widetilde{\mathfrak{H}} \supset \mathfrak{H}$ we put $\widetilde{\mathfrak{H}}_{0}:=\widetilde{\mathfrak{H}} \ominus \operatorname{mul} T_{\text {min }}$, so that

$$
\widetilde{\mathfrak{H}}=\operatorname{mul} T_{\min } \oplus \widetilde{\mathfrak{H}}_{0}
$$

It is clear that $\mathfrak{H}_{0} \subset \widetilde{\mathfrak{H}}_{0}\left(\right.$ for $\mathfrak{H}_{0}$ see $(53)$ ).

Let $\widetilde{T} \in \widetilde{\operatorname{Self}}_{0}\left(T_{\text {min }}\right)$ be a linear relation in a Hilbert space $\widetilde{\mathfrak{H}} \supset \mathfrak{H}$ and let $\widetilde{\mathfrak{H}}$ be decomposed as in (56) (for the class $\widetilde{\operatorname{Self}}_{0}$ see Definition 2.14). In the sequel we denote by $\widetilde{T}_{0}$ the operator part of $\widetilde{T}$. Since mul $\widetilde{T}=\operatorname{mul} T_{\min }$, it follows that $\widetilde{T}_{0}$ is a self-adjoint operator in $\widetilde{\mathfrak{H}}_{0}$. Let $E_{0}(\cdot)$ be the orthogonal spectral measure of $\widetilde{T}_{0}$ and let $F_{0}(\cdot): \mathbb{R} \rightarrow\left[\mathfrak{H}_{0}\right]$ be a distribution function given by

$$
F_{0}(t)=\widetilde{P}_{\mathfrak{H}_{0}} E_{0}((-\infty, t)) \uparrow \mathfrak{H}_{0}, \quad t \in \mathbb{R},
$$


where $\widetilde{P}_{\mathfrak{H}_{0}}$ is the orthoprojector in $\widetilde{\mathfrak{H}}_{0}$ onto $\mathfrak{H}_{0}$. It is clear that a spectral function $F(\cdot)$ of $T_{\text {min }}$ generated by $\widetilde{T}$ is of the form

$$
F(t)=\operatorname{diag}\left(F_{0}(t), 0\right): \mathfrak{H}_{0} \oplus \operatorname{mul} T_{\text {min }} \rightarrow \mathfrak{H}_{0} \oplus \operatorname{mul} T_{\text {min }} .
$$

Proposition 3.14. Let system (34) be definite. Then for each pseudospectral function $\Sigma(\cdot)$ of this system there exists a unique (up to the equivalence) exit space extension $\widetilde{T} \in \widetilde{\operatorname{Self}}_{0}\left(T_{\min }\right)$ such that the corresponding spectral function $F(\cdot)$ of $T_{\min }$ satisfies

$$
((F(\beta)-F(\alpha)) \widetilde{f}, \widetilde{f})_{\mathfrak{H}}=\int_{[\alpha, \beta)}(d \Sigma(s) \widehat{f}(s), \widehat{f}(s)), \quad \widetilde{f} \in \mathfrak{H}_{b}, \quad-\infty<\alpha<\beta<\infty .
$$

Moreover, if $\widetilde{T}$ is a linear relation in a Hilbert space $\widetilde{\mathfrak{H}} \supset \mathfrak{H}$, then there exists a unitary operator $\widetilde{V} \in\left[\widetilde{\mathfrak{H}}_{0}, L^{2}(\Sigma ; \mathbb{H})\right]$ such that $\widetilde{V} \uparrow \mathfrak{H}_{0}=V_{0, \Sigma}$ and the operators $\widetilde{T}_{0}$ and $\Lambda_{\Sigma}$ are unitarily equivalent by means of $\widetilde{V}$.

Proof. For a given pseudospectral function $\Sigma(\cdot)$ we put $L_{0}=V_{\Sigma} \mathfrak{H}_{0}$ and $L_{0}^{\perp}=L^{2}(\Sigma ; \mathbb{H}) \ominus L_{0}$, so that $L^{2}(\Sigma ; \mathbb{H})=L_{0} \oplus L_{0}^{\perp}$. Assume also that

$$
\widetilde{\mathfrak{H}}_{0}:=\mathfrak{H}_{0} \oplus L_{0}^{\perp}, \quad \widetilde{\mathfrak{H}}:=\operatorname{mul} T_{\min } \oplus \mathfrak{H}_{0} \oplus L_{0}^{\perp}=\operatorname{mul} T_{\min } \oplus \widetilde{\mathfrak{H}}_{0}
$$

and let $\widetilde{V} \in\left[\widetilde{\mathfrak{H}}_{0}, L^{2}(\Sigma ; \mathbb{H})\right]$ be a unitary operator given by

$$
\widetilde{V}=\left(V_{0, \Sigma}, I_{L_{0}^{\perp}}\right): \mathfrak{H}_{0} \oplus L_{0}^{\perp} \rightarrow L^{2}(\Sigma ; \mathbb{H}) .
$$

Since $\operatorname{ker} V_{\Sigma}=\operatorname{mul} T_{\text {min }}$, it follows that $\mathfrak{H}_{0}^{\prime}=\mathfrak{H}_{0}, \widetilde{\mathfrak{H}}_{0}^{\prime}=\widetilde{\mathfrak{H}}_{0}$ and $\widetilde{V}^{\prime}=\widetilde{V}$ (see 46), (47) and (48)). Therefore, by Lemma 3.5 identities 49 with $\widetilde{V}^{\prime}=\widetilde{V}$ define a self-adjoint operator $\widetilde{T}_{0}$ in $\mathfrak{H}_{0}$. Moreover, in view of 49 the operators $T_{0}$ and $\Lambda=\Lambda_{\Sigma}$ are unitarily equivalent by means of $\widetilde{V}$. Hence the spectral measure $E_{0}(\cdot)$ of $\widetilde{T}_{0}$ satisfies

$$
E_{0}([\alpha, \beta))=\widetilde{V}^{*} E_{\Sigma}([\alpha, \beta)) \widetilde{V}, \quad-\infty<\alpha<\beta<\infty .
$$

Observe also that $\widetilde{V} \mathfrak{H}_{0}=V_{\Sigma} \mathfrak{H}=L_{0}$ and by Proposition 3.13 operator $\Lambda_{\Sigma}$ is $L_{0}$-minimal. Therefore, the operator $\widetilde{T}_{0}$ is $\mathfrak{H}_{0}$-minimal.

It follows from the second identity in 60 that $\widetilde{T}:=\left(\{0\} \oplus \operatorname{mul} T_{\min }\right) \oplus \widetilde{T}_{0}$ is a selfadjoint linear relation in $\widetilde{\mathfrak{H}}$ with the operator part $\widetilde{T}_{0}$ and $\operatorname{mul} \widetilde{T}=\operatorname{mul} T_{\min }$. Moreover, $\{0\} \oplus \operatorname{mul} T_{\min } \subset T_{\min } \subset\left(T_{\min }\right)_{\widetilde{\mathfrak{H}}}^{*}$ and by Lemma $3.5 \widetilde{T}_{0} \subset\left(T_{\min }\right)_{\widetilde{\mathfrak{H}}}^{*}$. Hence $\widetilde{T} \subset\left(T_{\min }\right)_{\widetilde{\mathfrak{H}}}^{*}$ and, consequently, $T_{\min } \subset \widetilde{T}$. Observe also that relation $\widetilde{T}$ is $\mathfrak{H}$-minimal, since operator $\widetilde{T}_{0}$ is $\mathfrak{H}_{0^{-}}$ minimal. Hence $\widetilde{T} \in \widetilde{\operatorname{Self}}_{0}\left(T_{\text {min }}\right)$.

Next we assume that $F(\cdot)$ is a spectral function of $T_{\min }$ generated by $\widetilde{T}$ and let $F_{0}(\cdot)$ be given by (57). By using (62) and (61) one can easily show that

$$
F_{0}(\beta)-F_{0}(\alpha)=\widetilde{P}_{\mathfrak{H}_{0}} E_{0}([\alpha, \beta)) \uparrow \mathfrak{H}_{0}=V_{0, \Sigma}^{*} E_{\Sigma}([\alpha, \beta)) V_{0, \Sigma}, \quad-\infty<\alpha<\beta<\infty .
$$

Therefore, by (58) and (55) one has

$$
F(\beta)-F(\alpha)=V_{\Sigma}^{*} E_{\Sigma}([\alpha, \beta)) V_{\Sigma}, \quad-\infty<\alpha<\beta<\infty,
$$

which is equivalent to $(59)$. Finally, uniqueness of $\widetilde{T}$ directly follows from $(59)$ and $\mathfrak{H}$-minimality of $\widetilde{T}$.

The following corollary is immediate from Proposition 3.14 . 
Corollary 3.15. Let $\Sigma(\cdot)$ be a pseudospectral function of the definite system (34). Then $V_{0, \Sigma}$ is a unitary operator from $\mathfrak{H}_{0}$ onto $L^{2}(\Sigma ; \mathbb{H})$ if and only if $n_{+}\left(T_{\min }\right)=n_{-}\left(T_{\min }\right)$ and the corresponding extension $\widetilde{T}$ from Proposition 3.14 is canonical, that is $\widetilde{T} \in \operatorname{Self}_{0}\left(T_{\min }\right)$. If these conditions are satisfied, then operators $\widetilde{T}_{0}$ (the operator part of $\widetilde{T}$ ) and $\Lambda_{\Sigma}$ are unitarily equivalent by means of $V_{0, \Sigma}$.

Remark 3.16. Applying [2, Theorem 1] to the directing mapping (52) one can give another proof of Proposition 3.14 .

The following theorem is well known (see e.g. [27, 28, 29]).

Theorem 3.17. For each generalized resolvent $R(\lambda)$ of $T_{\min }$ there exists a unique operator function $\Omega(\cdot): \mathbb{C} \backslash \mathbb{R} \rightarrow[\mathbb{H}]$ such that for each $\tilde{f} \in L_{\Delta}^{2}(\mathcal{I})$ and $\lambda \in \mathbb{C} \backslash \mathbb{R}$

$$
R(\lambda) \widetilde{f}=\pi_{\Delta}\left(\int_{\mathcal{I}} Y_{0}(\cdot, \lambda)\left(\Omega(\lambda)+\frac{1}{2} \operatorname{sgn}(t-x) J\right) Y_{0}^{*}(t, \bar{\lambda}) \Delta(t) f(t) d t\right), \quad f \in \tilde{f} .
$$

Moreover, $\Omega(\cdot)$ is a Nevanlinna operator function.

Definition 3.18. [27, 29] The operator function $\Omega(\cdot)$ is called the characteristic matrix of the symmetric system (34) corresponding to the generalized resolvent $R(\lambda)$.

Remark 3.19. For a much more general situation formula (63) is obtained in [30, 31].

Since $\Omega(\cdot)$ is a Nevanlinna function, it follows that the identity (the Stieltjes formula)

$$
\Sigma_{\Omega}(s)=\lim _{\delta \rightarrow+0} \lim _{\varepsilon \rightarrow+0} \frac{1}{\pi} \int_{-\delta}^{s-\delta} \operatorname{Im} \Omega(\sigma+i \varepsilon) d \sigma .
$$

defines a distribution $[\mathbb{H}]$-valued function $\Sigma_{\Omega}(\cdot)$. This function is called a spectral function of $\Omega(\cdot)$.

Theorem 3.20. Assume that system (34) is absolutely definite. Let $\widetilde{T} \in \widetilde{\operatorname{Self}}_{0}\left(T_{\text {min }}\right)$, let $F(\cdot)$ and $R(\cdot)$ be the spectral function and the generalized resolvent of $T_{\min }$ respectively generated by $\widetilde{T}$, let $\Omega(\cdot)$ be the characteristic matrix corresponding to $R(\cdot)$ and let $\Sigma_{\Omega}(\cdot)$ be the spectral function of $\Omega(\cdot)$. Then $\Sigma(\cdot)=\Sigma_{\Omega}(\cdot)$ is a unique pseudospectral function of the system (34) such that (59) holds.

Proof. (1) Assume that $\widetilde{T}$ is a linear relations in the Hilbert space $\widetilde{\mathfrak{H}} \supset \mathfrak{H}$. By using 63 and the Stieltjes-Livšic inversion formula one proves identity $(59)$ for $\Sigma(\cdot)=\Sigma_{\Omega}(\cdot)$ in the same way as Theorem 4 in [29].

Next assume that $\mathfrak{H}$ and $\widetilde{\mathfrak{H}}$ are decomposed as in $(53)$ and $(56)$, respectively. Since $\operatorname{mul} \widetilde{T}=\operatorname{mul} T_{\min }$, it follows from $(59)$ and $(14)$ that for any $\widetilde{f} \in \mathfrak{H}_{b}$ one has $\widehat{f} \in \mathcal{L}^{2}(\Sigma ; \mathbb{H})$ and $\|\widehat{f}\|_{\mathcal{L}^{2}(\Sigma ; \mathbb{H})}=\left\|P_{\widetilde{\mathfrak{H}}_{0}} \widetilde{f}\right\|_{\widetilde{\mathfrak{H}}} \leqslant\|\widetilde{f}\|_{\mathfrak{H} \text {. }}$ Hence the operator $V_{b} \widetilde{f}:=\pi_{\Sigma} \widehat{f}, \quad \widetilde{f} \in \mathfrak{H}_{b}$, admits a continuation to an operator $V \in\left[\mathfrak{H}, L^{2}(\Sigma ; \mathbb{H})\right]$ satisfying

$$
\|V \widetilde{f}\|_{L^{2}(\Sigma ; \mathbb{H})}=\left\|P_{\widetilde{\mathfrak{H}}_{0}} \widetilde{f}\right\|_{\widetilde{\mathfrak{H}}}, \quad \widetilde{f} \in \mathfrak{H} .
$$

It follows from (65), (56) and the inclusion $\mathfrak{H}_{0} \subset \widetilde{\mathfrak{H}}_{0}$ that $V \widetilde{f}=0, \tilde{f} \in \operatorname{mul} T_{\min }$, and $\|V \widetilde{f}\|_{L^{2}(\Sigma ; \mathbb{H})}=\|\widetilde{f}\|_{\widetilde{\mathfrak{H}}}=\|\widetilde{f}\|_{\mathfrak{H}}, \tilde{f} \in \mathfrak{H}_{0}$. Thus $V$ is a partial isometry with $\operatorname{ker} V=\operatorname{mul} T_{\min }$ and, consequently, $\Sigma(\cdot)=\Sigma_{\Omega}(\cdot)$ is a pseudospectral function of the system (34) such that (59) holds.

(2) Next we show that each pseudospectral function $\Sigma(\cdot)$ satisfying $(59)$ coincides with $\Sigma_{\Omega}(\cdot)$. So, let $\Sigma(\cdot)$ be such a function, let $V_{\Sigma}$ be the corresponding Fourier transform and let $E_{\Sigma}$ be spectral measure (16). Then by (59) for each finite interval $\delta=[\alpha, \beta) \subset \mathbb{R}$ one has

$$
F(\beta)-F(\alpha)=V_{\Sigma}^{*} E_{\Sigma}(\delta) V_{\Sigma}
$$


and Proposition 3.4 yields

$$
(F(\beta)-F(\alpha)) \tilde{f}=\pi_{\Delta}\left(\int_{\delta} Y_{0}(\cdot, s) d \Sigma(s) \widehat{f}(s)\right), \quad \delta=[\alpha, \beta) \subset \mathbb{R}, \quad \widetilde{f} \in \mathfrak{H}_{b} .
$$

Substituting (42) into (67) and then using the Fubini theorem one can easily show that

$$
(F(\beta)-F(\alpha)) \widetilde{f}=\pi_{\Delta}\left(\int_{\mathcal{I}} K_{\delta, \Sigma}(\cdot, u) \Delta(u) f(u) d u\right), \quad \delta=[\alpha, \beta) \subset \mathbb{R}, \quad \tilde{f} \in \mathfrak{H}_{b}, \quad f \in \widetilde{f},
$$

where

$$
K_{\delta, \Sigma}(t, u)=\int_{\delta} Y_{0}(t, s) d \Sigma(s) Y_{0}^{*}(u, s), \quad t, u \in \mathcal{I}
$$

Let $K_{\delta, \Sigma_{\Omega}}(t, u)$ be given by 69 with $\Sigma(s)=\Sigma_{\Omega}(s)$ and let $K_{\delta}(t, u)=K_{\delta, \Sigma}(t, u)-K_{\delta, \Sigma_{\Omega}}(t, u)$, $t, u \in \mathcal{I}$. It follows from Theorem 2.7 that there exist a scalar measure $\sigma$ on $\mathcal{B}$ and functions $\Psi, \Psi_{\Omega}: \mathbb{R} \rightarrow[\mathbb{H}]$ such that

$$
\Sigma(\beta)-\Sigma(\alpha)=\int_{\delta} \Psi(s) d \sigma(s) \text { and } \Sigma_{\Omega}(\beta)-\Sigma_{\Omega}(\alpha)=\int_{\delta} \Psi_{\Omega}(s) d \sigma(s)
$$

for any finite $\delta=[\alpha, \beta)$. Let $\widetilde{\Psi}(s)=\Psi(s)-\Psi_{\Omega}(s)$. Then in view of 69 one has

$$
K_{\delta}(t, u)=\int_{\delta} Y_{0}(t, s) \widetilde{\Psi}(s) Y_{0}^{*}(u, s) d \sigma(s), \quad t, u \in \mathcal{I}, \quad \delta=[\alpha, \beta) \subset \mathbb{R} .
$$

Since $\Sigma_{\Omega}(\cdot)$ also satisfies $(59)$, identity $(68)$ holds with $K_{\delta, \Sigma_{\Omega}}$ in place of $K_{\delta, \Sigma}$. Hence

$$
\pi_{\Delta}\left(\int_{\mathcal{I}} K_{\delta}(\cdot, u) \Delta(u) f(u) d u\right)=0, \quad \delta=[\alpha, \beta) \subset \mathbb{R}, \quad f \in \mathcal{L}_{\Delta}^{2}(\mathcal{I}), \quad \pi_{\Delta} f \in \mathfrak{H}_{b} .
$$

Denote by $F\left(F^{\prime}\right)$ the set of all finite intervals $\delta=[\alpha, \beta) \subset \mathbb{R}\left(\right.$ resp. $\left.\delta^{\prime}=\left[\alpha^{\prime}, \beta^{\prime}\right) \subset \mathcal{I}\right)$ with rational endpoints. Moreover, let $\left\{e_{j}\right\}_{1}^{n}$ be a basis in $\mathbb{H}$. It follows from 72 that for any $\delta \in F, \delta^{\prime} \in F^{\prime}$ and $e_{j}$ there exists a Borel set $B=B\left(\delta, \delta^{\prime}, e_{j}\right) \subset \mathcal{I}$ such that $\mu_{1}(\mathcal{I} \backslash B)=0$ and

$$
\int_{\delta^{\prime}} \Delta(t) K_{\delta}(t, u) \Delta(u) e_{j} d u=0, \quad t \in B
$$

(here $\mu_{1}$ is the Lebesgue measure on $\mathcal{I}$ ). For each $\delta \in F$ put

$$
\widetilde{K}_{\delta}(t, u)=\Delta(t) K_{\delta}(t, u) \Delta(u)=\int_{\delta} \Delta(t) Y_{0}(t, s) \widetilde{\Psi}(s) Y_{0}^{*}(u, s) \Delta(u) d \sigma(s)
$$

and let $B_{\delta}=\left\{\{t, u\} \in \mathcal{I} \times \mathcal{I}: \widetilde{K}_{\delta}(t, u)=0\right\}, B_{0}=\bigcap_{\delta \in F} B_{\delta}$. It follows from 73at that $\mu_{2}\left(\mathcal{I} \times \mathcal{I} \backslash B_{\delta}\right)=0, \delta \in F$, and hence $\mu_{2}\left(\mathcal{I} \times \mathcal{I} \backslash B_{0}\right)=0$ (here $\mu_{2}$ is the Lebesgue measure on $\mathcal{I} \times \mathcal{I})$. Let $X_{\Delta}=\{t \in \mathcal{I}: \Delta(t)$ is invertible $\}$. Since system (34) is absolutely definite, it follows that $\mu_{1}\left(X_{\Delta}\right)>0$. Hence $\mu_{2}\left(X_{\Delta} \times X_{\Delta}\right)>0$ and, consequently, $\left(X_{\Delta} \times X_{\Delta}\right) \cap B_{0} \neq \emptyset$. Therefore, there exist $t_{0}$ and $u_{0}$ in $I$ such that the operators $\Delta\left(t_{0}\right)$ and $\Delta\left(u_{0}\right)$ are invertible and the identity

$$
\widetilde{K}_{\delta}\left(t_{0}, u_{0}\right)=\int_{\delta} \Delta\left(t_{0}\right) Y_{0}\left(t_{0}, s\right) \widetilde{\Psi}(s) Y_{0}^{*}\left(u_{0}, s\right) \Delta\left(u_{0}\right) d \sigma(s)=0
$$

holds for all $\delta \in F$. Hence $\Delta\left(t_{0}\right) Y_{0}\left(t_{0}, s\right) \widetilde{\Psi}(s) Y_{0}^{*}\left(u_{0}, s\right) \Delta\left(u_{0}\right)=0(\sigma$-a.e. on $\mathbb{R})$ and invertibility of $Y_{0}\left(t_{0}, s\right)$ and $Y_{0}^{*}\left(u_{0}, s\right)$ yields $\widetilde{\Psi}(s)=0(\sigma$-a.e. on $\mathbb{R})$. Thus, $\Psi(s)=\Psi_{\Omega}(s)$ and by 70 $\Sigma(s)=\Sigma_{\Omega}(s)$.

Now combining Proposition 3.14, Theorem 3.20 and Corollary 3.15 we arrive at the following theorem. 
Theorem 3.21. Let system (34) be absolutely definite. Then:

(1) Identities (63) and (64) give a bijective correspondence $\Sigma(\cdot)=\Sigma_{\widetilde{T}}(\cdot)$ between all extensions $\widetilde{T} \in \widetilde{\operatorname{Self}}_{0}\left(T_{\min }\right)$ and all pseudospectral functions $\Sigma(\cdot)$. More precisely, let $\widetilde{T} \in \widetilde{\operatorname{Self}}_{0}\left(T_{\min }\right)$, let $R(\cdot)=R_{\widetilde{T}}(\cdot)$ be the generalized resolvent of $T_{\min }$ induced by $\widetilde{T}$, let $\Omega(\cdot)=\Omega_{\widetilde{T}}(\cdot)$ be the characteristic matrix corresponding to $R_{\widetilde{T}}(\cdot)$ and let $\Sigma_{\widetilde{T}}(\cdot)$ be the spectral function of $\Omega_{\widetilde{T}}(\cdot)$. Then $\Sigma_{\widetilde{T}}(\cdot)$ is a pseudospectral function of system (34). And vice versa, for each pseudospectral function $\Sigma(\cdot)$ of system (34) there exists a unique (up to equivalence) $\widetilde{T} \in \widetilde{\operatorname{Self}}_{0}\left(T_{\min }\right)$ such that $\Sigma(\cdot)=\Sigma_{\widetilde{T}}(\cdot)$.

(2) If $\widetilde{T} \in \widetilde{\operatorname{Self}}_{0}\left(T_{\min }\right)$ and $\Sigma(\cdot)=\Sigma_{\widetilde{T}}(\cdot)$, then operators $\widetilde{T}_{0}$ (the operator part of $\widetilde{T}$ ) and $\Lambda_{\Sigma}$ are unitarily equivalent and hence they have the same spectral properties. In particular this implies that the spectral multiplicity of $\widetilde{T}_{0}$ does not exceed $\operatorname{dim} \mathbb{H}$.

(3) $V_{0, \Sigma}$ is a unitary operator from $\mathfrak{H}_{0}$ onto $L^{2}(\Sigma ; \mathbb{H})$ if and only if $n_{+}\left(T_{\min }\right)=n_{-}\left(T_{\min }\right)$ and $\Sigma(\cdot)=\Sigma_{\widetilde{T}}$ with $\widetilde{T} \in \operatorname{Self}_{0}\left(T_{\min }\right)$. In this case the operators $\widetilde{T}_{0}$ and $\Lambda_{\Sigma}$ are unitarily equivalent by means of $V_{0, \Sigma}$.

Next, combining the results of this subsection with Assertion 3.10 one gets the following theorem.

Theorem 3.22. The set of spectral functions of system (34) is not empty if and only if $\operatorname{mul} T_{\min }=\{0\}$. If this condition is satisfied, then the sets of spectral and pseudospectral functions of system (34) coincide and hence Proposition 3.14, Theorems 3.20, 3.21 and Corollary 3.15 are valid for spectral functions (instead of pseudospectral ones). Moreover, in this case statements of Proposition 3.14 and Theorem 3.21 hold with $\widetilde{T}$ and $V_{\Sigma}$ in place of $\widetilde{T}_{0}$ and $V_{0, \Sigma}$ respectively.

Remark 3.23. For a not necessarily absolutely definite system Theorem 3.21 could be easily obtained from Theorem 1 in [2] applied to directing mapping (52). For this purpose it would be needed one of the statements of the mentioned Theorem 1, which is not proved in 2] (namely, uniqueness of a spectral function $V$ of $\langle S ; \Phi\rangle$ for a given extension $\widetilde{S}=\widetilde{S}^{*}$ of $S$, where the notations are taken from [2]). In fact, we do not know whether Theorem 3.21 is valid for not absolutely definite systems.

\section{Parametrization of PSeudospectral And SPeCtral FunCtions}

Proposition 4.1. [5] Let system (34) be definite and let $n_{-}\left(T_{\min }\right) \leqslant n_{+}\left(T_{\min }\right)$. Then: (1) There exist a finite dimensional Hilbert space $\widetilde{\mathcal{H}}_{b}$, a subspace $\mathcal{H}_{b} \subset \widetilde{\mathcal{H}}_{b}$ and a surjective linear mapping

$$
\Gamma_{b}=\left(\Gamma_{0 b}, \widehat{\Gamma}_{b}, \Gamma_{1 b}\right)^{\top}: \operatorname{dom} \mathcal{T}_{\max } \rightarrow \widetilde{\mathcal{H}}_{b} \oplus \widehat{H} \oplus \mathcal{H}_{b}
$$

such that for all $y, z \in \operatorname{dom} \mathcal{T}_{\max }$ the following identity is valid

$$
[y, z]_{b}=\left(\Gamma_{0 b} y, \Gamma_{1 b} z\right)-\left(\Gamma_{1 b} y, \Gamma_{0 b} z\right)+i\left(P_{\mathcal{H}_{b}^{\perp}} \Gamma_{0 b} y, P_{\mathcal{H}_{b}^{\perp}} \Gamma_{0 b} z\right)+i\left(\widehat{\Gamma}_{b} y, \widehat{\Gamma}_{b} z\right)
$$

(here $\mathcal{H}_{b}^{\perp}=\widetilde{\mathcal{H}}_{b} \ominus \mathcal{H}_{b}$ ).

(2) If $\Gamma_{b}$ is a surjective linear mapping (75) satisfying (76), then a collection $\Pi_{+}=\left\{\mathcal{H}_{0} \oplus \mathcal{H}_{1}, \Gamma_{0}, \Gamma_{1}\right\}$ with

$$
\begin{gathered}
\mathcal{H}_{0}=H \oplus \widehat{H} \oplus \widetilde{\mathcal{H}}_{b}=H_{0} \oplus \widetilde{\mathcal{H}}_{b}, \quad \mathcal{H}_{1}=H \oplus \widehat{H} \oplus \mathcal{H}_{b}=H_{0} \oplus \mathcal{H}_{b} \\
\Gamma_{0}\{\widetilde{y}, \widetilde{f}\}=\left\{-y_{1}(a), i\left(\widehat{y}(a)-\widehat{\Gamma}_{b} y\right), \Gamma_{0 b} y\right\} \in H \oplus \widehat{H} \oplus \widetilde{\mathcal{H}}_{b} \\
\Gamma_{1}\{\widetilde{y}, \widetilde{f}\}=\left\{y_{0}(a), \frac{1}{2}\left(\widehat{y}(a)+\widehat{\Gamma}_{b} y\right),-\Gamma_{1 b} y\right\} \in H \oplus \widehat{H} \oplus \mathcal{H}_{b}
\end{gathered}
$$


is a boundary triplet for $T_{\max }$ (in $(78)$ and $(79) y \in \operatorname{dom} \mathcal{T}_{\max }$ is a function corresponding to $\{\widetilde{y}, \widetilde{f}\} \in T_{\max }$ in accordance with Remark 3.12, (2)).

If in addition $n_{+}\left(T_{\min }\right)=n_{-}\left(T_{\min }\right)$, then

$$
\widetilde{\mathcal{H}}_{b}=\mathcal{H}_{b}, \quad \mathcal{H}_{0}=\mathcal{H}_{1}=: \mathcal{H}=H_{0} \oplus \mathcal{H}_{b}
$$

and $\Pi_{+}$turns into an ordinary boundary triplet $\Pi=\left\{\mathcal{H}, \Gamma_{0}, \Gamma_{1}\right\}$ for $T_{\max }$ with $\mathcal{H}$ defined by the second identity in 80 .

The boundary triplet $\Pi_{+}$constructed in Proposition 4.1 is called a decomposing boundary triplet for $T_{\max }$.

Below we suppose that the following assumptions are satisfied:

(A1) System (34) is absolutely definite and $n_{-}\left(T_{\text {min }}\right) \leqslant n_{+}\left(T_{\text {min }}\right)$

(A2) $\widetilde{\mathcal{H}}_{b}$ and $\mathcal{H}_{b}\left(\subset \widetilde{\mathcal{H}}_{b}\right)$ are finite dimensional Hilbert spaces and $\Gamma_{b}$ is a surjective linear mapping (75) satisfying (76).

(A3) $\mathcal{H}_{0}$ and $\mathcal{H}_{1}$ are finite dimensional Hilbert spaces 77

(A4) $\Pi_{+}=\left\{\mathcal{H}_{0} \oplus \mathcal{H}_{1}, \Gamma_{0}, \Gamma_{1}\right\}$ is the decomposing boundary triplet (78), (79) for $T_{\max }$ and $M_{+}(\cdot)$ is the Weyl function of $\Pi_{+}$in the sense of Definition 2.11 .

Definition 4.2. A boundary parameter $\tau$ is a collection $\tau=\left\{\tau_{+}, \tau_{-}\right\} \in \widetilde{R}_{+}\left(\mathcal{H}_{0}, \mathcal{H}_{1}\right)$ of the form (18).

In the case of equal deficiency indices $n_{+}\left(T_{\min }\right)=n_{-}\left(T_{\min }\right)$ identities 80$)$ hold and a boundary parameter is an operator pair $\tau \in \widetilde{R}(\mathcal{H})$ defined by $(19)$. If in addition $\tau \in \widetilde{R}^{0}(\mathcal{H})$, then a boundary parameter will be called self-adjoint. In this case $\tau$ admits the representation as a self-adjoint operator pair $(20)$.

It follows from Theorem 2.15 that for each boundary parameter $\tau=\left\{\tau_{+}, \tau_{-}\right\}$defined by (18) there exist the limits $\mathcal{B}_{\tau}$ and $\mathcal{B}_{\tau}$ of the form 28 and 29 .

Definition 4.3. A boundary parameter $\tau$ will be called admissible if $\mathcal{B}_{\tau}=\widehat{\mathcal{B}}_{\tau}=0$.

The following proposition is immediate from the results of [6].

Proposition 4.4. (i) If $\lim _{y \rightarrow \infty} \frac{1}{i y} M_{+}(i y)\left\lceil\mathcal{H}_{1}=0\right.$, then the boundary parameter $\tau$ is admissible if and only if $\mathcal{B}_{\tau}=0$.

(ii) Every boundary parameter is admissible if and only if mul $T_{\min }=\operatorname{mul} T_{\max }$ (see Assertion 3.2. (2)) or equivalently, if and only if $\lim _{y \rightarrow \infty} \frac{1}{i y} M_{+}(i y) \uparrow \mathcal{H}_{1}=0$ and

$$
\lim _{y \rightarrow+\infty} y\left(\operatorname{Im}\left(M_{+}(i y) h_{0}, h_{0}\right)_{\mathcal{H}_{0}}+\frac{1}{2}\left\|P_{2} h_{0}\right\|^{2}\right)=+\infty, \quad h_{0} \in \mathcal{H}_{0}, \quad h_{0} \neq 0,
$$

where $P_{2}$ is the orthoprojector in $\mathcal{H}_{0}$ onto $\mathcal{H}_{2}=\mathcal{H}_{0} \ominus \mathcal{H}_{1}$.

In the following theorem we describe all pseudospectral functions of the system (34) in terms of the boundary parameter $\tau$.

Theorem 4.5. Let the assumptions (A1)-(A4) be satisfied. Moreover, let

$$
M_{+}(\lambda)=\left(\begin{array}{cc}
m_{0}(\lambda) & M_{2+}(\lambda) \\
M_{3+}(\lambda) & M_{4+}(\lambda)
\end{array}\right): \underbrace{H_{0} \oplus \widetilde{\mathcal{H}}_{b}}_{\mathcal{H}_{0}} \rightarrow \underbrace{H_{0} \oplus \mathcal{H}_{b}}_{\mathcal{H}_{1}}, \quad \lambda \in \mathbb{C}_{+}
$$


be the block-matrix representation of the Weyl function $M_{+}(\cdot)$ and let

$$
\begin{gathered}
\Omega_{0}(\lambda)=\left(\begin{array}{cc}
m_{0}(\lambda) & -\frac{1}{2} I_{H, H_{0}} \\
-\frac{1}{2} P_{H_{0}, H} & 0
\end{array}\right): \underbrace{H_{0} \oplus H}_{\mathbb{H}} \rightarrow \underbrace{H_{0} \oplus H}_{\mathbb{H}}, \quad \lambda \in \mathbb{C} \backslash \mathbb{R} \\
S_{1}(\lambda)=\left(\begin{array}{cc}
m_{0}(\lambda)-\frac{i}{2} P_{\widehat{H}} & M_{2+}(\lambda) \\
-P_{H_{0}, H} & 0
\end{array}\right): \underbrace{H_{0} \oplus \widetilde{\mathcal{H}}_{b}}_{\mathcal{H}_{0}} \rightarrow \underbrace{H_{0} \oplus H}_{\mathbb{H}}, \quad \lambda \in \mathbb{C}_{+} \\
S_{2}(\lambda)=\left(\begin{array}{cc}
m_{0}(\lambda)+\frac{i}{2} P_{\widehat{H}} & -I_{H, H_{0}} \\
M_{3+}(\lambda) & 0
\end{array}\right): \underbrace{H_{0} \oplus H}_{\mathbb{H}} \rightarrow \underbrace{H_{0} \oplus \mathcal{H}_{b}}_{\mathcal{H}_{1}}, \quad \lambda \in \mathbb{C}_{+},
\end{gathered}
$$

where $P_{H_{0}, H} \in\left[H_{0}, H\right]$ is the orthoprojector in $H_{0}$ onto $H, I_{H, H_{0}} \in\left[H, H_{0}\right]$ is the embedding operator of $H$ into $H_{0}$ and $P_{\widehat{H}} \in\left[H_{0}\right]$ is the orthoprojector in $H_{0}$ onto $\widehat{H}$ (see (33)). Then the identity

$$
\Omega_{\tau}(\lambda)=\Omega_{0}(\lambda)+S_{1}(\lambda)\left(C_{0}(\lambda)-C_{1}(\lambda) M_{+}(\lambda)\right)^{-1} C_{1}(\lambda) S_{2}(\lambda), \quad \lambda \in \mathbb{C}_{+}
$$

together with the Stieltjes inversion formula (9) establishes a bijective correspondence between all admissible boundary parameters $\tau=\left\{\tau_{+}, \tau_{-}\right\}$defined by (18) and all pseudospectral functions $\Sigma(\cdot)=\Sigma_{\tau}(\cdot)$ of the system (34). Moreover, statement of the theorem is valid for arbitrary (not necessarily admissible) boundary parameters $\tau$ if and only if $\lim _{y \rightarrow \infty} \frac{1}{i y} M_{+}(i y) \uparrow \mathcal{H}_{1}=0$ and identity (81) is satisfied.

Proof. Application of Theorem 2.12 to the decomposing boundary triplet $\Pi_{+}$for $T_{\max }$ shows that the boundary problem (21)-(23) with operators $\Gamma_{0}$ and $\Gamma_{1}$ of the form $(78)$ and $(79)$ gives a parametrization $R(\lambda)=R_{\tau}(\lambda)$ of all generalized resolvents of $T_{\min }$ by means of a boundary parameter $\tau$. Denote by $\widetilde{T}^{\tau}\left(\in \widetilde{\operatorname{Self}}\left(T_{\min }\right)\right)$ the extension of $T_{\min }$ generating $R_{\tau}(\cdot)$ and by $\Omega_{\tau}(\cdot)\left(=\Omega_{\widetilde{T}^{\tau}}(\cdot)\right)$ the characteristic matrix corresponding to $R_{\tau}(\cdot)$. Clearly, the identities $\widetilde{T}=\widetilde{T}^{\tau}$ and $\Omega(\cdot)=\Omega_{\tau}(\cdot)$ give a parametrization of all extensions $\widetilde{T} \in \widetilde{\operatorname{Self}}\left(T_{\min }\right)$ and all characteristic matrices $\Omega(\cdot)$ by means of a boundary parameter $\tau$. Moreover, representation (84) of $\Omega_{\tau}(\cdot)$ was obtained in [32, Theorem 4.6]. Observe also that according to Theorem 2.15 $T^{\tau} \in \widetilde{\operatorname{Self}}_{0}\left(T_{\text {min }}\right)$ if and only if $\tau$ is admissible. Combining these facts with Theorem 3.21 we arrive at the first statement of the theorem. The second statement is implied by the first one and Proposition 4.4 .

Remark 4.6. The entries of the matrix in 82 can be defined in terms of boundary values of respective operator solutions of (34) at the endpoints $a$ and $b$ (for more details see [5, Proposition 4.5]).

Assume now that $T_{\min }$ has equal deficiency indices $n_{+}\left(T_{\min }\right)=n_{-}\left(T_{\min }\right)$. Then identities $(28)$ and (29) take a simpler form (31) and (32), where $M(\cdot)$ is the Weyl function of an (ordinary) decomposing boundary triplet $\Pi$ for $T_{\max }$.

Theorem 4.7. Let in addition to the assumptions of Theorem 4.5 the identity $n_{+}\left(T_{\min }\right)=n_{-}\left(T_{\min }\right)$ holds. Moreover, let

$$
M(\lambda)=\left(\begin{array}{ll}
m_{0}(\lambda) & M_{2}(\lambda) \\
M_{3}(\lambda) & M_{4}(\lambda)
\end{array}\right): \underbrace{H_{0} \oplus \mathcal{H}_{b}}_{\mathcal{H}} \rightarrow \underbrace{H_{0} \oplus \mathcal{H}_{b}}_{\mathcal{H}}, \quad \lambda \in \mathbb{C} \backslash \mathbb{R}
$$

be the block-matrix representation of the Weyl function $M(\cdot)$, let $\Omega_{0}(\lambda)$ be given by (83) and let

$$
S(\lambda)=\left(\begin{array}{cc}
m_{0}(\lambda)-\frac{i}{2} P_{\widehat{H}} & M_{2}(\lambda) \\
-P_{H_{0}, H} & 0
\end{array}\right): \underbrace{H_{0} \oplus \mathcal{H}_{b}}_{\mathcal{H}} \rightarrow \underbrace{H_{0} \oplus H}_{\mathbb{H}}, \quad \lambda \in \mathbb{C} \backslash \mathbb{R} .
$$


Then the identity

$$
\Omega_{\tau}(\lambda)=\Omega_{0}(\lambda)+S(\lambda)\left(C_{0}(\lambda)-C_{1}(\lambda) M(\lambda)\right)^{-1} C_{1}(\lambda) S^{*}(\bar{\lambda}), \quad \lambda \in \mathbb{C} \backslash \mathbb{R}
$$

together with the Stieltjes formula (9) establishes a bijective correspondence between all admissible boundary parameters $\tau$ of the form (19) and all pseudospectral functions $\Sigma(\cdot)=\Sigma_{\tau}(\cdot)$ of system (34). Moreover, $V_{0, \Sigma}\left(\in\left[\mathfrak{H}_{0}, L^{2}(\Sigma ; \mathbb{H})\right]\right)$ is a unitary operator if and only if $\Sigma(\cdot)=\Sigma_{\tau}(\cdot)$ with a self-adjoint (admissible) boundary parameter $\tau$.

The above statements are valid for arbitrary (not necessarily admissible) boundary parameters $\tau$ if and only if

$$
\lim _{y \rightarrow \infty} \frac{1}{i y} M(i y)\left\lceil\mathcal{H}_{1}=0 \text { and } \lim _{y \rightarrow \infty} y \cdot \operatorname{Im}(M(i y) h, h)=+\infty, \quad h \in \mathcal{H}, \quad h \neq 0 .\right.
$$

Proof. According to [32, Theorem 4.9] in the case $n_{+}\left(T_{\min }\right)=n_{-}\left(T_{\min }\right)$ identity (84) admits representation (85). Combining of this fact with Theorem 4.5 and Theorem 3.21, (3) yields the required statements.

The following corollary is immediate from Theorem 3.22 .

Corollary 4.8. If $\operatorname{mul} T_{\min }=\{0\}$, then Theorems 4.5 and 4.7 are valid for spectral functions $\Sigma(\cdot)$ (instead of pseudospectral ones).

\section{BIBLIOGRAPHY}

1. I.S. Kats. Linear relations generated by the canonical differential equation of phase dimension 2, and eigenfunction expansion // St. Petersburg Math. J. 14, 429-452 (2003).

2. H. Langer and B. Textorius. Spectral functions of a symmetric linear relation with a directing mapping, I // Proc. Roy. Soc. Edinburgh Sect. A 97, 165-176 (1984).

3. A.L. Sakhnovich, L.A. Sakhnovich, and I.Ya. Roitberg, Inverse problems and nonlinear evolution equations. Solutions, Darboux matrices and Weyl-Titchmarsh functions, De Gruyter Studies in Mathematics 47. De Gruyter, Berlin (2013).

4. D.Z. Arov, H. Dym. Bitangential direct and inverse problems for systems of integral and differential equations, Encyclopedia of mathematics and its applications. Cambridge University Press, Cambridge (2012).

5. S. Albeverio, M.M. Malamud, V.I.Mogilevskii. On Titchmarsh-Weyl functions and eigenfunction expansions of first-order symmetric systems // Integr. Equ. Oper. Theory. 77:3, 303-354 (2013) .

6. V.I.Mogilevskii. On exit space extensions of symmetric operators with applications to first order symmetric systems // Methods Funct. Anal. Topology 19:3, 268-292 (2013).

7. H. Langer and B. Textorius. Spectral functions of a symmetric linear relation with a directing mapping, II // Proc. Roy. Soc. Edinburgh Sect. A. 101:1-2, 111-124(1985).

8. V. M. Bruk. On a class of boundary value problems with spectral parameter in the boundary condition // Math. USSR-Sb. 29:2, 186-192 (1976).

9. V. M. Bruk. Extensions of symmetric relations, Math. Notes 22:6, 953-958 (1977).

10. V.A. Derkach, M.M. Malamud. Generalized resolvents and the boundary value problems for Hermitian operators with gaps // J. Funct. Anal. 95:1, 1-95 (1991).

11. V.A. Derkach, S. Hassi, M.M. Malamud, H.S.V. de Snoo. Generalized resolvents of symmetric operators and admissibility // Methods Funct. Anal. Topol. 6:3, 24-55 (2000).

12. V.I. Gorbachuk, M.L. Gorbachuk, Boundary problems for differential-operator equations, Kluver Acad. Publ., Dordrecht-Boston-London (1991). (Russian edition: Naukova Dumka, Kiev (1984)).

13. A.N. Kochubei. Extensions of symmetric operators and symmetric binary relations // Math. Notes. 17:1, 25-28 (1975).

14. M. M. Malamud. On the formula of generalized resolvents of a nondensely defined Hermitian operator // Ukr. Math. Zh. 44:12, 1658-1688 (1992). 
15. V. Khrabustovskyi. Expansion in eigenfunctions of relations generated by pair of operator differential expressions // Methods Funct. Anal. Topology 15:2, 137-151 (2009).

16. V. Khrabustovskyi. Eigenfunction expansions associated with an operator differential equation nonlinearly depending on a spectral parameter // Methods Funct. Anal. Topol. 20:1, 68-91 (2014).

17. N. Dunford and J.T. Schwartz. Linear operators. Part2. Spectral theory, Interscience Publishers, New York-London (1963).

18. I.S. Kats. On Hilbert spaces generated by monotone Hermitian matrix-functions // Khar'kov. Gos. Univ. Uchen. Zap. 34, 95-113 (1950). Zap.Mat.Otdel.Fiz.-Mat. Fak. i Khar'kov. Mat. Obshch. 22:4 95-113 (1950).

19. V.I. Mogilevskii. Nevanlinna type families of linear relations and the dilation theorem // Methods Funct. Anal. Topol. 12:1, 38-56 (2006).

20. V.I. Mogilevskii. Description of generalized resolvents and characteristic matrices of differential operators in terms of the boundary parameter // Math. Notes. 90:4, 548-570 (2011).

21. V.I. Mogilevskii. Boundary triplets and Krein type resolvent formula for symmetric operators with unequal defect numbers // Methods Funct. Anal. Topol. 12:3, 258-280 (2006).

22. V.A. Derkach, S. Hassi, M.M. Malamud, and H.S.V. de Snoo, Boundary relations and generalized resolvents of symmetric operators // Russian J. Math. Ph. 16:1, 17-60 (2009).

23. V.M. Bruk. On a number of linearly independent quadratically integrable solutions of systems of differential equations // Functional Analysis. Ul'yanovsk Pedagogical Institute. 5. 25-33 (1975).

24. M. Lesch, M.M. Malamud. On the deficiency indices and self-adjointness of symmetric Hamiltonian systems // J. Diff. Equat. 189:2, 556-615 (2003).

25. V. Mogilevskii. Boundary pairs and boundary conditions for general (not necessarily definite) firstorder symmetric systems with arbitrary deficiency indices // Math. Nachr. 285:14-15, 1895-1931 (2012).

26. B.C. Orcutt. Canonical differential equations. PhD thesis, Univ. of Virginia (1969).

27. V.M. Bruk. Linear relations in a space of vector functions, // Math. Notes. 24:4, 767-773 (1978).

28. A. Dijksma, H. Langer, H.S.V. de Snoo. Eigenvalues and pole functions of Hamiltonian systems with eigenvalue depending boundary conditions // Math. Nachr. 161:1, 107-153 (1993).

29. A.V. Straus. On generalized resolvents and spectral functions of differential operators of an even order // Izv. Akad. Nauk. SSSR, Ser.Mat. 21:6, 785-808 (1957).

30. V.I. Khrabustovsky. On the characteristic operators and projections and on the solutions of Weyl type of dissipative and accumulative operator systems. General case // J. Math. Phys. Anal. Geom. 2:2, 149-175 (2006).

31. V.M. Bruk. On the characteristic operator of an integral equation with a nevanlinna measure in the infinite-dimensional case // J. Math. Phys. Anal. Geom. 10:2, 163-188 (2014).

32. V.I.Mogilevskii. On generalized resolvents and characteristic matrices of first-order symmetric systems // Methods Funct. Anal. Topol. 20:4, 328-348 (2014).

Vadim Iosifovich Mogilevskii,

Department of Differential Equations, Bashkir State University,

32 Zaki Validi,

Ufa, 450076, Russia

E-mail: vadim.mogilevskii@gmail.com 